\title{
El efecto de la movilidad durante los estudios sobre el desajuste educativo de los graduados recientes. Evidencia a partir de la encuesta de inserción laboral de los titulados y tituladas de las universidades catalanas*
}

\author{
Antonio Di Paolo \\ Raúl Ramos \\ AQR, Universitat de Barcelona
}

\section{Resumen}

Desde 1987, año de su creación, hasta el curso 2016/17, más de medio millón de estudiantes universitarios españoles han participado en los programas Erasmus y Erasmus + de intercambio de estudiantes entre los distintos Estados miembros de la Unión Europea, así como en el Programa SICUE/Séneca que fomenta la movilidad entre universidades españolas y que lleva funcionando desde el año 2000. Algo más de una cuarta parte de estos participantes cursaban sus estudios universitarios en universidades catalanas. Sin embargo, a partir de la literatura internacional, no existe un claro consenso sobre los efectos de la realización de estancias formativas y prácticas en el extranjero en distintos indicadores laborales de los graduados en su transición desde la universidad hacia el mercado laboral. El objetivo de este trabajo es ofrecer nueva evidencia sobre el efecto de la movilidad nacional e internacional por motivos de estudios o laborales en una dimensión poco analizada hasta el momento pero muy relevante en el contexto español: el desajuste educativo. Distintos trabajos han mostrado las dificultades de los graduados universitarios españoles para conseguir un empleo acorde a su formación, un emparejamiento que podría verse facilitado por las competencias adquiridas durante la movilidad de estudios y prácticas. A partir de la información disponible en las encuestas de inserción laboral de los titulados y tituladas en las universidades catalanas (más de 30.000 observaciones) se analiza la asociación entre haber realizado alguna forma de movilidad nacional o internacional y la sobrecualificación autopercibida en el empleo cuatro años después de acabar los estudios universitarios. Los resultados obtenidos muestran una asociación positiva entre ambas variables que podría estar relacionada con la adquisición de determinadas competencias durante las estancias, que permiten a estos graduados asumir algunas funciones en su puesto de trabajo en mejores condiciones que sus compañeros de promoción.

Palabras clave: movilidad académica, movilidad laboral, calidad del empleo, sobreeducación.

Clasificación JEL: I23, I26, J61.

* Los autores desean agradecer los comentarios y sugerencias recibidos de los editores de la revista y de un evaluador anónimo, así como a AQU Catalunya por la cesión de los datos utilizados en el estudio. También agradecen el soporte económico del proyecto del Programa Estatal de Fomento de la I+D+i orientada a los Retos de la Sociedad, convocatoria 2016 (ECO2016-75805-R). 


\begin{abstract}
Since 1987, year of its creation, until the academic year 2016/17, more than half million of Spanish university students have participated in the Erasmus and Erasmus+ Exchange programmes between the different European Union member states as well as in the program SICUE/Seneca Programme that boosts mobility between Spanish universities since 2000. More than a fourth part of the participants in these programmes were students of Catalan universities. However, the international literature has not reached a clear consensus on the effects of academic mobility and mobility linked to internships on labour market outcomes during transition from the university to work. The aim of this article is to offer new evidence on the impact of national and international mobility by reasons of studies or labour in a dimension that has received little attention in literature but that it is very relevant in the Spanish context: educational mismatches. Distinct works have shown the difficulties of Spanish graduates to get a job in line with their training, a match that could be facilitated by the competences acquired during the mobility experience. Using the available information from the survey on Employment Outcomes of Graduates from Catalan universities (more than 30,000 observations), we analyse the association between having experienced some form of national or international mobility during studies and self-perceived overeducation in employment four years after graduation. The obtained results show a positive association between both variables that could be related to the acquisition of some competences, which allow these graduates to assume some functions in their workplace in better conditions that their mates.
\end{abstract}

Keywords: academic mobility, labour mobility, job quality, overeducation.

JEL classification: I23, I26, J61

\title{
1. Introducción y objetivos
}

Realizar estancias temporales en otros países durante la realización de los estudios es hoy en día una opción muy común entre los jóvenes europeos. Desde 1987, año de su creación, hasta el curso 2016/17, más de tres millones de estudiantes han participado en los programas Erasmus y Erasmus + de intercambio de estudiantes entre los distintos Estados miembros de la Unión Europea, medio millón de los cuales eran españoles. A nivel nacional, el Programa SICUE, que lleva funcionando desde el año 2000, también ha facilitado la movilidad entre centros universitarios españoles de miles de estudiantes, aunque no en todos los cursos se han convocado las becas Séneca asociadas a dicho programa. Si bien es cierto que la participación en este tipo de programas es en sí misma una experiencia enriquecedora e inolvidable, también existe una opinión muy generalizada de que permite mejorar los resultados laborales de los participantes (European Commission, 2014).

Sin embargo, no existe un claro consenso sobre cuáles son los efectos de la realización de estancias formativas y de prácticas en el extranjero sobre la transición de los graduados desde la universidad hacia el mercado laboral, ni sobre los mecanismos que explicarían esos mejores resultados. Por un lado, la teoría del capital humano explica la decisión de realizar estudios en el extranjero como parte de una inversión orientada a aumentar las competencias y mejorar las habilidades de los participantes, especialmente en el ámbito del conocimiento de idiomas, mejoras que se traducirían en mayores niveles de productividad y, por tanto, de empleo y salarios (Becker, 1964). 
Por otro lado, la teoría de la señalización argumenta que la participación en este tipo de actividades no aumenta necesariamente la productividad de los individuos, sino que las empresas identifican la experiencia de movilidad internacional como una señal de determinadas características de los participantes que, frecuentemente, no son observables de manera directa (Spence, 1973). Tal y como señalan Rospigliosi et al. (2014), ambas teorías no son necesariamente excluyentes, sino que ofrecen justificaciones complementarias sobre el posible efecto positivo de la movilidad sobre los resultados laborales que contrasta, sin embargo, con la escasa evidencia favorable encontrada en la literatura internacional (Di Pietro, 2014; Waibel et al., 2017).

Teniendo en cuenta estos antecedentes, el objetivo del trabajo es analizar el efecto de la movilidad internacional durante los estudios en una dimensión poco considerada hasta el momento pero muy relevante en el contexto español: el desajuste educativo. De hecho, distintos trabajos han mostrado las dificultades de los graduados universitarios españoles para conseguir un empleo acorde a su formación (véase, por ejemplo, Murillo y Raymond, 2017), un emparejamiento que podría verse facilitado por las competencias adquiridas durante la movilidad de estudios y prácticas.

A partir de la información disponible en las encuestas de inserción laboral de los titulados y tituladas en las universidades catalanas (más de 30.000 observaciones) se analiza la asociación entre haber realizado movilidad nacional e internacional por razones de estudios o laborales y la sobrecualificación autopercibida en el empleo obtenido cuatro años después de finalizar los estudios universitarios. Los resultados obtenidos muestran una asociación positiva entre ambas variables que podría estar relacionada con la adquisición de determinadas competencias durante las estancias de movilidad que permiten a estos graduados asumir algunas funciones en su puesto de trabajo en mejores condiciones que sus compañeros de promoción.

El resto del trabajo se organiza en cuatro apartados. A continuación, se realiza un breve resumen de la literatura que ha analizado la relación entre la movilidad internacional y los resultados laborales, así como el mecanismo a través del cual puede tener efectos sobre el desajuste educativo. El tercer apartado describe los datos utilizados en el análisis y la metodología aplicada. El cuarto apartado presenta los resultados obtenidos y, por último, el quinto apartado resume las principales conclusiones, así como las limitaciones del estudio.

\section{Revisión de la literatura}

Hay un amplio número de trabajos que han analizado el efecto de la movilidad durante los estudios sobre resultados laborales. Son de especial interés en el ámbito de este trabajo los relacionados con los efectos de participar en distintos programas de movilidad, pero especialmente aquellos referidos a programas de movilidad internacional y más concretamente al programa ERASMUS (Teichler y Janson, 2007; Parey y Waldinger, 2011; Di Pietro, 2012; Rodrigues, 2013; European Commission, 2014; Di Pietro, 2015; entre otros). 
Estos estudios han permitido identificar las ventajas potenciales para los participantes en estos programas. En concreto, los graduados que participan en programas de movilidad internacional tienen una mejor situación en el mercado de trabajo que los que no han participado, como consecuencia de haber adquirido distintas competencias especialmente bien valoradas por las empresas (Di Pietro, 2014): en primer lugar, la mejora en el conocimiento de idiomas extranjeros; en segundo lugar, la capacidad para desenvolverse de manera adecuada en entornos multiculturales; en tercer lugar, una mayor disposición a la movilidad internacional en un entorno laboral, y por último, una mayor flexibilidad y capacidad para adaptarse a nuevas situaciones, así como una mayor madurez y confianza en las propias habilidades.

Ahora bien, pese a la existencia de estas ventajas potenciales y, tal y como se ha comentado en la introducción, no existe un claro consenso sobre cuáles son los efectos de la movilidad durante los estudios sobre los resultados laborales tras la graduación.

Waibel et al. (2017) realizan una revisión sistemática de los estudios previos centrando su interés en aquellos que han analizado la dimensión vertical de los resultados laborales, es decir, aquellos que analizan la probabilidad de estar empleado y el salario recibido, y descartando aquellos que analizan otras características del empleo como, por ejemplo, conseguir un trabajo en un país distinto al de origen. Para ello, identifican aquellos estudios que miden el efecto de la movilidad a través de indicadores objetivos de aquellos otros que se centran en indicadores subjetivos. En lo que se refiere a los indicadores objetivos, prácticamente todos los estudios concluyen que la movilidad internacional durante los estudios no afecta de manera significativa a la probabilidad de estar empleado ni reduce el tiempo en encontrar un primer empleo al finalizar los estudios. En cambio, sí que se encuentran evidencias a favor de la existencia de un mayor nivel salarial entre aquellos graduados con experiencia internacional algunos años después de la graduación. Con respecto a los indicadores subjetivos, un aspecto que destacan varios estudios es el efecto positivo de la movilidad sobre el proceso de maduración personal y la mayor confianza en sí mismos que experimentan los participantes en dichos programas. Sin embargo, dichos autores también concluyen que los efectos de la movilidad sobre la carrera profesional solo emergen si se dan unas circunstancias favorables para ello. En concreto, la literatura ha encontrado evidencia favorable al papel de diferentes factores moderadores como el género, el entorno familiar y el estatus socioeconómico, el área de estudio y la situación del mercado de trabajo en el país de origen (Di Pietro, 2014).

Focalizándose específicamente en estos factores moderadores, Van Mol (2017) introduce además en el análisis la perspectiva de los empleadores, un aspecto que no había sido considerado hasta ese momento de manera generalizada debido, principalmente, a las deficiencias en la información estadística disponible. En concreto, Van Mol (2017) utiliza información procedente del Flash Eurobarometer 304 «Employers’ perception of graduate employability» con una muestra de más de 7.000 empresas de más de 50 empleados ubicadas en los países miembros de la Unión Europea en 2010, así como en Islandia, Noruega y Turquía. En este sentido, es importante destacar que este autor no 
solo considera la movilidad internacional vinculada a estudios sino también a otras experiencias laborales. Los resultados de su análisis muestran que solo uno de cada cinco empleadores encuestados consideran la movilidad internacional durante los estudios como importante, si bien asignan una mayor relevancia a la realización de prácticas internacionales. En concreto, uno de cada cuatro empleadores encuestados lo consideran un factor relevante a la hora de contratar a un graduado reciente y en algunos países este porcentaje es claramente superior situándose por encima del 40 por 100.

Existe también un amplio número de trabajos que han considerado la relación existente entre la movilidad geográfica de los trabajadores y el desajuste educativo. Distintos autores entre los cuales cabe citar a Büchel y Van Ham (2003), Hensen et al. (2009) o Ramos y Sanromá (2013) han demostrado que la dimensión del mercado de trabajo en el que los individuos buscan empleo reduce la probabilidad de estar sobreeducado ya que permite un mejor emparejamiento entre vacantes y trabajadores. Iammarino y Marinelli (2015), Crescenzi et al. (2016) y Kazakis y Faggian (2017) muestran también cómo las decisiones de migración de los graduados reducen la probabilidad de estar sobreeducado. Sin embargo, hasta donde conocemos, no existe evidencia previa sobre la relación existente entre estancias cortas en el extranjero vinculadas a estudios o a prácticas y el desajuste educativo en el mercado de trabajo local ${ }^{1}$.

Hasta donde conocemos, Iriondo-Múgica (2017) es el único estudio que ha evaluado el efecto de la participación de los universitarios españoles en el programa Erasmus tanto en lo que se refiere a empleo como a salarios. En concreto, este autor analiza dos muestras de estudiantes de licenciatura representativas del conjunto del sistema universitario español (la Encuesta de Inserción Laboral de Titulados Universitarios 2014 elaborada por el INE y la Encuesta de Inserción Laboral 2008 de la Universidad Complutense de Madrid). Sus resultados muestran que, entre cinco y seis años después de terminar los estudios, la movilidad internacional tiene un efecto positivo sobre los salarios, pero no sobre la probabilidad de estar ocupado ni sobre el tiempo en encontrar el primer empleo.

\section{Datos y metodología}

El análisis que se realiza a continuación está basado en las encuestas de inserción laboral a los titulados y tituladas de las universidades catalanas, que se lleva a cabo por la Agencia para la Calidad del Sistema Universitario de Cataluña (AQU). Estas encuestas se realizan cada tres años desde el año 2001 y se dirigen a todos los es-

${ }^{1}$ También existe una amplia literatura que relaciona la existencia de sobreeducación entre los inmigrantes como el resultado de la falta de compatibilidad del capital humano adquirido en sus países de origen con los requerimientos del mercado de trabajo del país de destino y, en suma, de su imperfecta transferibilidad internacional (FRIEDBERG, 2000; SANROMÁ et al., 2015). Sin embargo, esta no sería la situación considerada en este trabajo dado que se trata de estancias cortas y, además, el título es emitido por las universidades del país de origen. 
tudiantes universitarios graduados cuatro años antes en las universidades catalanas tanto públicas como privadas. Nuestro análisis se limita a las cuatro últimas olas (2008, 2011, 2014 y 2017 -graduados en 2004, 2007, 2010 y 2013) y a los graduados de las siete universidades públicas para los cuales es posible obtener información homogénea para las variables relevantes en nuestro análisis.

Las encuestas realizadas por AQU contienen información académica relevante sobre los titulados universitarios, así como un amplio número de características socio-demográficas y de sus resultados laborales cuatro años después de la graduación. Parte de esta información es facilitada por el encuestado, mientras que algunos datos académicos se obtienen directamente de registros administrativos. Pueden obtenerse más detalles sobre la metodología de realización de la encuesta así como sobre la información disponible en las distintas olas en AQU Catalunya (2017)².

Además de limitar el análisis a las cuatro últimas olas y a los graduados/as de las universidades públicas catalanas que se encuentran empleados/as en el momento de realizar la encuesta (más del 90 por 100), se ha excluido del análisis a los residentes en el extranjero (alrededor del 3 por 100), a aquellos individuos que no habían variado de empleo a raíz de la obtención del título universitario en el momento de realizar la encuesta, las observaciones que presentan missing values de las variables de interés y, por último, a los graduados en medicina dadas las peculiaridades de esta profesión en relación al resto de titulaciones universitarias consideradas ${ }^{3}$. La muestra obtenida para el conjunto de las cuatro olas está formada por 30.265 individuos, alrededor de 8.000 para cada una de las olas consideradas.

En cuanto a la primera de las variables de interés de nuestro estudio, la realización de parte de los estudios universitarios o bien el desempeño de experiencias laborales (que pueden estar vinculadas a la universidad de origen como prácticas curriculares) en un país extranjero, la información facilitada por la encuesta permite identificar a aquellos graduados que han tenido una o varias experiencias de movilidad internacional o nacional ${ }^{4}$ durante sus estudios y saber cuál ha sido la finalidad: solo académica, solo laboral o académica y laboral. Tal y como se puede observar en el Cuadro 1, el porcentaje de individuos que han realizado alguna experiencia de movilidad entre los años considerados en nuestro análisis se acerca al 37 por 100 en el total del período analizado, con valores que fluctúan entre el 32 por 100 en 2008 y el 40 por 100 en 2014.

2 Para más información consulten el siguiente enlace válido en noviembre de 2018: http://www.aqu.cat/ estudis/ilaboral_2014.html.

3 Más concretamente, como los titulados en medicina tendencialmente están realizando el período de especialización tras egresar de la universidad (el llamado MIR, Médicos Internos Residentes), las cohortes de titulación no coinciden con las de los titulados de otros ámbitos.

${ }^{4}$ Cabe destacar que solo en las últimas dos olas de la encuesta es posible distinguir entre movilidad nacional e internacional, mientras que solo en la encuesta del 2017 se pregunta explícitamente sobre el país de destino. Por tanto, en este trabajo se hace referencia a episodios de movilidad sin distinguir entre destinos nacionales e internacionales. También parece interesante remarcar que en las últimas dos olas de la encuesta las experiencias de movilidad internacional representan alrededor del 28 por 100 de la totalidad de los casos; considerando solo los episodios de movilidad académica, resulta que aproximadamente el 78 por 100 representan estancias en otros países. 


\section{CUADRO 1}

PORCENTAJE DE ESTUDIANTES EN FUNCIÓN DE SU PARTICIPACIÓN EN ACTIVIDADES DE MOVILIDAD

\begin{tabular}{|l|r|r|r|r|r|}
\hline & \multicolumn{1}{|c|}{ Total } & \multicolumn{1}{|c|}{2008} & \multicolumn{1}{|c|}{2011} & \multicolumn{1}{|c|}{2014} & \multicolumn{1}{|c|}{2017} \\
\hline No movilidad & 63,01 & 68,03 & 61,19 & 59,50 & 62,22 \\
Movilidad académica & 17,55 & 13,68 & 17,02 & 21,44 & 19,05 \\
Movilidad laboral & 11,13 & 11,89 & 12,19 & 10,77 & 9,60 \\
Movilidad académica y laboral & 8,31 & 6,40 & 9,61 & 8,29 & 9,13 \\
Total & 100,00 & 100,00 & 100,00 & 100,00 & 100,00 \\
\hline
\end{tabular}

FUENTE: Elaboración propia a partir de las Encuestas de Inserción Laboral (AQU).

La otra variable relevante en nuestro análisis es la relativa al desajuste educativo. En concreto, la información facilitada por la encuesta permite medir el desajuste educativo desde una perspectiva subjetiva ${ }^{5}$. En concreto, el individuo encuestado expresa su opinión sobre los requisitos asociados al trabajo desempeñado y las competencias requeridas para desarrollar las funciones asociadas, a partir de las cuales se han generado las siguientes cuatro categorías:

1. No se requiere ningún título universitario para desempeñar el trabajo realizado ni se realizan funciones asociadas a las propias de un grado universitario.

2. Se requiere titulación universitaria, pero las funciones desempeñadas no son las propias de la titulación.

3. Se requiere titulación universitaria específica, pero las funciones desempeñadas no son las propias de dicha titulación específica del encuestado.

4. Se realizan las funciones propias de la titulación específica del encuestado.

De la definición de las diferentes categorías se desprende que pueden relacionarse con distintas situaciones de desajuste educativo, tanto desde una perspectiva vertical como horizontal. Mientras que la primera categoría se refiere claramente a una situación de sobreeducación, la segunda y la tercera se asemejan mucho más a situaciones de desajuste horizontal mientras que únicamente la cuarta se corresponde a un emparejamiento adecuado entre el graduado y el empleo que desempeña.

Tal y como se puede observar en el Cuadro 2, el porcentaje de trabajadores sobreeducados se sitúa alrededor del 11,5 por 100 en el conjunto del período analizado oscilando entre valores próximos al 9 por 100 al principio del periodo, pero con una tendencia claramente creciente superando el 15 por 100 en 2014 y reduciéndose ligeramente hasta el 13 por 100 en 2017. En lo que se refiere a las situaciones de desajuste horizontal, su incidencia se sitúa en valores cercanos al 8 por 100 durante todo el período analizado. Así pues, el desajuste educativo afecta durante el período

5 McGUINESS et al. (2018) describen los distintos métodos propuestos en la literatura para la medición del desajuste educativo y ofrecen una panorámica sobre los estudios recientes en este ámbito. 


\section{CUADRO 2}

\section{PORCENTAJE DE ESTUDIANTES EN FUNCIÓN DE LA ADECUACIÓN ENTRE LOS ESTUDIOS REALIZADOS Y LA ACTIVIDAD LABORAL DESEMPEÑADA}

\begin{tabular}{|l|c|c|c|c|c|}
\hline & Total & $\mathbf{2 0 0 8}$ & $\mathbf{2 0 1 1}$ & $\mathbf{2 0 1 4}$ & $\mathbf{2 0 1 7}$ \\
\hline $\begin{array}{l}\text { Ningún requisito, funciones no } \\
\text { universitarias }\end{array}$ & 11,46 & 9,39 & 8,78 & 15,67 & 12,81 \\
$\begin{array}{l}\text { Requisito de titulación universi- } \\
\text { taria, funciones no propias }\end{array}$ & 3,73 & 3,99 & 3,98 & 4,37 & 2,70 \\
$\begin{array}{l}\text { Requisito de titulación especí- } \\
\text { fica, funciones no propias de la } \\
\text { titulación específica }\end{array}$ & 4,80 & 5,44 & 5,36 & 2,72 & 5,25 \\
$\begin{array}{l}\text { Funciones propias de la titula- } \\
\text { ción específica (independiente- } \\
\text { mente del requisito) }\end{array}$ & 80,02 \\
Total & 81,18 & 81,88 & 77,25 & 79,24 \\
\hline
\end{tabular}

FUENTE: Elaboración propia a partir de las Encuestas de Inserción Laboral (AQU).

analizado a más del 20 por 100 de los graduados, un valor ligeramente inferior al encontrado por otros estudios recientes como Davia et al. (2017), si bien es cierto que dichos autores utilizan el método estadístico y no el subjetivo y realizan su análisis para las siete regiones NUTS16 y no para comunidades autónomas españolas.

Para poder analizar si existe alguna relación entre las variables consideradas, se estima un modelo probit ordenado donde la variable endógena es el desajuste educativo en el orden indicado anteriormente. Los regresores de interés son un conjunto de indicadores derivados de una variable categórica (con categorías mutualmente excluyentes y que, por tanto, se considerarán en el análisis de manera conjunta) que recoge los distintos tipos de experiencias de movilidad: movilidad de estudios, movilidad de prácticas y ambas, siendo la categoría base la no movilidad. Sin embargo, y tal y como se ha comentado en el apartado anterior, también es necesario tener en cuenta el posible papel de distintos factores moderadores que pueden estar relacionados tanto con la propensión a llevar a cabo alguna experiencia de movilidad como con la probabilidad de tener un trabajo que no se ajusta a las cualificaciones y competencias adquiridas con los estudios. Con la finalidad de apreciar mejor el efecto mediador de distintas variables de control, se procede con la inclusión secuencial de las mismas en el modelo de regresión. Concretamente, en la especificación inicial (a) se incluyen solo variables sociodemográficas (género, edad cuadrática y nivel educativo de los padres) y académicas (cualificación media obtenida en el grado cursado, área de conocimiento distinguiendo humanidades, ciencias sociales, ciencias experimentales, ciencias de la salud y disciplinas técnicas, universidad y año de

\footnotetext{
${ }^{6}$ La subdivisión geográfica NUTS1 para España contempla siete áreas geográficas: noroeste, noreste, Comunidad de Madrid, centro, este, sur y Canarias.
} 
titulación). Sucesivamente (b) se añaden diferentes características laborales como la localización de la empresa (Barcelona, Girona, Lleida, Tarragona u otra CCAA), la titularidad pública o privada de la misma, el tipo de jornada (a tiempo completo o parcial) y los años de antigüiedad, el tamaño de la empresa y el sector de actividad (26 sectores). En la tercera etapa (c) se incluyen también las funciones desempeñadas en el puesto de trabajo, considerando categorías no mutualmente exclusivas (es decir, un individuo puede realizar más de una función a la vez). Los resultados obtenidos al estimar por máxima verosimilitud los diferentes modelos probit ordenados se presentan en el siguiente apartado. Cabe destacar que se obtienen resultados cualitativa y cuantitativamente similares utilizando diferentes modelos probit para cada uno de los valores de la medida de desajuste educativo utilizada, así como con un modelo lineal estimado por mínimos cuadrados ordinarios. No obstante, la capacidad de ajuste del probit ordenado y la precisión de las estimaciones es mayor frente a estas otras alternativas.

\section{Resultados}

Las estimaciones obtenidas para las tres especificaciones del modelo probit ordenado que explica el desajuste educativo en función de las experiencias de movilidad y de otras características de control se muestran en el Cuadro $3^{7}$.

Los coeficientes de las variables de control de la especificación inicial (columna a), indican que las mujeres tituladas tienen, a igualdad de otras características sociodemográficas y académicas, una menor propensión al desajuste educativo en sus puestos de trabajo, mientras que la edad incrementa el riesgo de desajuste con un efecto marginal decreciente. Los titulados cuyos padres tienen estudios universitarios (especialmente si ambos completaron estudios terciarios) tienen un menor desajuste educativo que los demás. Este resultado posiblemente se debe a las mejores redes sociales a las que pueden acceder familias con un bagaje educativo más favorable, que pueden favorecer una búsqueda de trabajo más eficiente. Asimismo, obtener una mayor nota final en la titulación reduce el desajuste educativo, debido tanto a efectos de señalización asociados al expediente académico, como a las mayores habilidades cognitivas y no cognitivas de los estudiantes que obtienen mejores notas. Con respecto a las áreas de estudio, se observa cómo relativamente a los titulados en disciplinas humanísticas, los que se titulan en el ámbito de salud presentan un mejor ajuste educativo, que podría explicarse en base a la relevancia de la regulación laboral de las profesiones asociadas a estas disciplinas. No obstante, también los titulados en ciencias sociales, en disciplinas técnicas y, en menor medida, en ciencias experimentales tienen una menor propensión a estar desajustados en el mercado laboral cuatro años después de graduarse.

\footnotetext{
${ }^{7}$ En el Cuadro 3 solo se muestran los coeficientes de las variables más relevantes, mientras que los resultados completos se detallan en la tabla del Anexo 2.
} 
En cuanto a la variable de interés, las experiencias de movilidad, las estimaciones que incluyen como variables de control solamente las características sociodemográficas y académicas no muestran diferencias significativas en la propensión al desajuste educativo entre los que no realizaron ninguna movilidad y los titulados que llevaron a cabo algún tipo de movilidad académica o laboral. Sin embargo, los diferentes grupos pueden tener distintas características laborales, por lo que en la columna (b) del Cuadro 3 se procede con la inclusión del conjunto de variables que capturan las características (observadas) de los trabajos de los titulados de la muestra. Una vez se controla por todas estas características, se evidencia una correlación condicional positiva entre las diferentes experiencias de movilidad de los titulados. Concretamente, los coeficientes estimados indican que tanto la movilidad durante los estudios (que captura prevalentemente las estancias realizadas bajo los programas de movilidad internacional y nacional mencionados anteriormente) como las experiencias laborales en ámbitos geográficos distintos al de la residencia habitual (bajo programas de prácticas o realizadas de forma individual) y las combinaciones de las dos formas mejoran el ajuste laboral. Entonces, manteniendo constantes tanto las características sociodemográficas y académicas, así como diferentes factores que caracterizan los trabajos obtenidos por los titulados, emerge un efecto beneficioso de las diferentes experiencias de movilidad nacional e internacional realizada por los titulados de las universidades públicas catalanas. Estos resultados también indican que, en cierta medida, la movilidad académica y laboral está relacionada con las diferentes variables laborales incluidas en el modelo.

Con respecto a estas variables, en las tablas con las estimaciones completas (Anexo 2) se puede observar cómo trabajar en empresas públicas, a tiempo completo, en empresas de más de diez trabajadores y tener una mayor antigüedad en la empresa también se relacionan positivamente con el ajuste educativo. Además, el tipo de contrato también influye en el desajuste educativo: los titulados que se constituyen como autónomos/empresarios tienen una mayor propensión al ajuste educativo que los que tienen un contrato indefinido en una empresa, lo que indica una predisposición de los primeros a desarrollar actividades que se ajustan a los estudios, en los que pueden aprovechar las competencias adquiridas. En cambio, los que se encuentran en situaciones contractuales inestables, especialmente los becarios, presentan un mayor desajuste en el mercado del trabajo. También existen diferencias condicionales en el desajuste educativo según sector de actividad, como muestran los coeficientes de los distintos indicadores de actividad económica.

En la tercera especificación, se añaden diferentes indicadores que capturan las funciones realizadas en el puesto de trabajo, que pueden explicar en buena medida diferencias en el desajuste educativo percibido de los titulados universitarios y, al mismo tiempo, estar relacionadas con las experiencias de movilidad. Como se muestra en la columna (c) del Cuadro 3, al añadir estos indicadores en el modelo que explica el desajuste educativo, los coeficientes asociados a los diferentes indicadores de movilidad reducen su valor, aunque la dirección y la significatividad de los efectos queda cualitativamente invariada. Este último resultado sugiere que los 
titulados que llevan a cabo experiencias de movilidad académica o laboral obtienen puestos de trabajo en los que pueden desarrollar funciones específicas que encajan con las competencias adquiridas durante los estudios universitarios. A partir de esta especificación, también resulta interesante destacar algunos resultados obtenidos en relación a las estimaciones de los coeficientes de las variables dicotómicas referentes al año de la encuesta. En concreto, el análisis de esta información permite obtener alguna indicación respecto a los cambios en la incidencia del desajuste educativo relacionados con la crisis económica y la sucesiva recuperación. Concretamente, con referencia al año base (encuesta del 2008, que se refiere a los titulados en 2004), se puede observar cómo el desajuste educativo (una vez controlado el efecto del resto de variables consideradas en el análisis) ha aumentado a lo largo de los años, pero la intensidad del incremento se ha ido reduciendo entre 2014 y 2017. Estos resultados posiblemente se deben a que durante la fase recesiva los empleadores tenían disponible un conjunto más amplio de potenciales trabajadores para ocupar las (relativamente pocas) plazas vacantes $\mathrm{y}$, por eso, tendían a seleccionar titulados universitarios, aunque las ofertas de trabajo no requiriesen estudios específicos o competencias concretas. En cambio, con el comienzo de la recuperación económica (que se observa entre los titulados de la última cohorte, que acabaron los estudios en 2013), este efecto negativo sobre el ajuste en el mercado del trabajo tiende a mitigarse.

Para obtener una perspectiva cuantitativa de la relación entre las variables de interés y el ajuste educativo, a partir de la última especificación con todas las variables de control se calculan los efectos marginales promedios (es decir, el promedio de los efectos marginales calculados para cada observación) de las distintas variables. ${ }^{8}$ Los efectos marginales de variables seleccionadas sobre la probabilidad de tener cada valor posible de la variable dependiente se reportan en el Cuadro 4 y evidencian que la movilidad aumenta la probabilidad de estar en el valor más elevado del indicador de ajuste educativo (4) cuando se realizan las funciones propias de la titulación específica del encuestado respecto a las otras posibilidades. El efecto marginal es ligeramente mayor para la combinación de experiencias laborales y académicas $(+1,7$ puntos porcentuales, p.p., frente a no movilidad) que para experiencias puramente académicas $(+1,2$ p.p.) o laborales ( $+1,3$ p.p.). Además, también se puede observar cómo llevar a cabo funciones de dirección ( $+6,4$ p.p.), enseñanza y asistencia técnica (+11 p.p.), investigación y desarrollo (+10 p.p.) y funciones técnicas $(+8,6$ p.p.) mejora la propensión al ajuste educativo, mientras realizar otras funciones (especialmente si son no cualificadas) tiene un efecto penalizador sobre el encaje entre las competencias de los titulados y sus puestos de trabajo.

${ }^{8}$ Para las variables discretas, el efecto marginal correspondiente se calcula como la diferencia en la probabilidad predicha cuando cada indicador se cambia de 0 a 1 . 


\section{CUADRO 3 \\ ESTIMACIÓN DEL MODELO PROBIT ORDENADO PARA EL DESAJUSTE EDUCATIVO}

\begin{tabular}{|c|c|c|c|}
\hline & (a) & (b) & (c) \\
\hline No movilidad & \multicolumn{3}{|c|}{ Categoría de referencia } \\
\hline Movilidad académica & $\begin{array}{c}0,026 \\
(0,023)\end{array}$ & $\begin{array}{c}0,070 * * * \\
(0,024)\end{array}$ & $\begin{array}{c}0,054 * * \\
(0,024)\end{array}$ \\
\hline Movilidad laboral & $\begin{array}{c}0,041 \\
(0,027)\end{array}$ & $\begin{array}{c}0,089 * * * \\
(0,028)\end{array}$ & $\begin{array}{c}0,058^{* *} \\
(0,029)\end{array}$ \\
\hline Movilidad académica y laboral & $\begin{array}{c}0,045 \\
(0,030)\end{array}$ & $\begin{array}{c}0,105^{* * *} \\
(0,032)\end{array}$ & $\begin{array}{r}0,076^{* *} \\
(0,033)\end{array}$ \\
\hline Mujer & $\begin{array}{c}0,040 * * \\
(0,018)\end{array}$ & $\begin{array}{c}0,017 \\
(0,019)\end{array}$ & $\begin{array}{c}0,043 * * \\
(0,020)\end{array}$ \\
\hline Edad & $\begin{array}{c}-0,095 * * * \\
(0,019)\end{array}$ & $\begin{array}{c}-0,096 * * * \\
(0,020)\end{array}$ & $\begin{array}{c}-0,102 * * * \\
(0,020)\end{array}$ \\
\hline Edad al cuadrado & $\begin{array}{c}0,001 * * * \\
(0,000)\end{array}$ & $\begin{array}{c}0,001 * * * \\
(0,000)\end{array}$ & $\begin{array}{c}0,001 * * * \\
(0,000)\end{array}$ \\
\hline Padres con estudios primarios o sin estudios & \multicolumn{3}{|c|}{ Categoría de referencia } \\
\hline Uno de los dos padres con estudios medios & $\begin{array}{l}-0,003 \\
(0,026)\end{array}$ & $\begin{array}{c}0,009 \\
(0,027)\end{array}$ & $\begin{array}{c}0,013 \\
(0,028)\end{array}$ \\
\hline Los dos padres con estudios medios & $\begin{array}{c}0,038 \\
(0,024)\end{array}$ & $\begin{array}{c}0,049 * * \\
(0,025)\end{array}$ & $\begin{array}{c}0,052 * * \\
(0,025)\end{array}$ \\
\hline Uno de los dos padres con estudios superiores & $\begin{array}{r}0,058^{* *} \\
(0,024)\end{array}$ & $\begin{array}{c}0,073 * * * \\
(0,025)\end{array}$ & $\begin{array}{c}0,062 * * \\
(0,025)\end{array}$ \\
\hline Los dos padres con estudios superiores & $\begin{array}{c}0,159 * * * \\
(0,025)\end{array}$ & $\begin{array}{c}0,153 * * * \\
(0,026)\end{array}$ & $\begin{array}{c}0,128 * * * \\
(0,027)\end{array}$ \\
\hline Nota final aprobado & \multicolumn{3}{|c|}{ Categoría de referencia } \\
\hline Nota final notable & $\begin{array}{c}0,187 * * * \\
(0,017)\end{array}$ & $\begin{array}{c}0,129 * * * \\
(0,018)\end{array}$ & $\begin{array}{c}0,088 * * * \\
(0,019)\end{array}$ \\
\hline Nota final sobresaliente & $\begin{array}{c}0,381 * * * \\
(0,055)\end{array}$ & $\begin{array}{c}0,251^{* * *} \\
(0,057)\end{array}$ & $\begin{array}{c}0,172 * * * \\
(0,058)\end{array}$ \\
\hline Nota final matrícula de honor & $\begin{array}{c}0,647 * * * \\
(0,143)\end{array}$ & $\begin{array}{c}0,489 * * * \\
(0,152)\end{array}$ & $\begin{array}{c}0,423 * * \\
(0,164)\end{array}$ \\
\hline Área de humanidades & \multicolumn{3}{|c|}{ Categoría de referencia } \\
\hline Área de ciencias sociales & $\begin{array}{c}0,492 * * * \\
(0,027)\end{array}$ & $\begin{array}{c}0,440 * * * \\
(0,029)\end{array}$ & $\begin{array}{c}0,370 * * * \\
(0,030)\end{array}$ \\
\hline Área de ciencias experimentales & $\begin{array}{c}0,387 * * * \\
(0,035)\end{array}$ & $\begin{array}{c}0,300 * * * \\
(0,038)\end{array}$ & $\begin{array}{c}0,116 * * * \\
(0,040)\end{array}$ \\
\hline Área de ciencias de la salud & $\begin{array}{c}0,828 * * * \\
(0,037)\end{array}$ & $\begin{array}{c}0,725 * * * \\
(0,047)\end{array}$ & $\begin{array}{c}0,532 * * * \\
(0,051)\end{array}$ \\
\hline Área de disciplinas técnicas & $\begin{array}{c}0,603 * * * \\
(0,036)\end{array}$ & $\begin{array}{c}0,634 * * * \\
(0,041)\end{array}$ & $\begin{array}{c}0,370 * * * \\
(0,043)\end{array}$ \\
\hline Universidad & Sí & Sí & Sí \\
\hline Año de la encuesta & Sí & Sí & Sí \\
\hline
\end{tabular}

FUENTE: Elaboración propia a partir de las Encuestas de Inserción Laboral (AQU).

NOTA: Los errores estándar (en paréntesis) son robustos a la heterocedasticidad. * significativo al 10 por 100 ** Significativo al 5 por 100. $* * *$ Significativo al 1 por 100. Los resultados completos correspondientes a la estimación de estos modelos se recogen en el Anexo 1. 


\section{CUADRO 3 (Continuación) \\ ESTIMACIÓN DEL MODELO PROBIT ORDENADO PARA EL DESAJUSTE EDUCATIVO}

\begin{tabular}{|l|c|c|c|}
\hline & (a) & (b) & (c) \\
\hline Provincia/CCAA de trabajo & No & Sí & Sí \\
\hline Características del empleo y de la empresa & No & Sí & Sí \\
\hline Funciones desempeñadas & No & No & Sí \\
\hline cut1 & $-2,280^{* * *}$ & $-1,884^{* * *}$ & $-2,167 * * *$ \\
& $(0,314)$ & $(0,352)$ & $(0,347)$ \\
cut2 & $-2,099 * * *$ & $-1,679 * * *$ & $-1,940 * * *$ \\
& $(0,314)$ & $(0,352)$ & $(0,347)$ \\
cut3 & $-1,905^{* * *}$ & $-1,466 * * *$ & $-1,708^{* * *}$ \\
& $(0,314)$ & $(0,352)$ & $(0,347)$ \\
\hline Número de observaciones & 30.265 & 30.265 & 30.265 \\
\hline
\end{tabular}

FUENTE: Elaboración propia a partir de las Encuestas de Inserción Laboral (AQU).

NOTA: Los errores estándar (en paréntesis) son robustos a la heterocedasticidad. * Significativo al 10 por 100 , ** Significativo al 5 por 100 . *** Significativo al 1 por 100 . Los resultados completos correspondientes a la estimación de estos modelos se recogen en el Anexo 1.

\section{CUADRO 4}

\section{EFECTOS MARGINALES DEL MODELO PROBIT ORDENADO PARA EL DESAJUSTE EDUCATIVO}

\begin{tabular}{|l|c|c|c|c|}
\hline & $(\mathbf{1})$ & $\mathbf{( 2 )}$ & $\mathbf{( 3 )}$ & $\mathbf{( 4 )}$ \\
\hline Movilidad académica & $-0,008^{* *}$ & $-0,002^{* *}$ & $-0,002^{* *}$ & $0,012^{* *}$ \\
Movilidad laboral & $(0,004)$ & $(0,001)$ & $(0,001)$ & $(0,005)$ \\
& $-0,009^{* *}$ & $-0,002^{* *}$ & $-0,002^{* *}$ & $0,013^{* *}$ \\
& $(0,004)$ & $(0,001)$ & $(0,001)$ & $(0,006)$ \\
& $-0,011^{* *}$ & $-0,003^{* *}$ & $-0,003^{* *}$ & $0,017 * *$ \\
& $(0,005)$ & $(0,001)$ & $(0,001)$ & $(0,007)$ \\
\hline Mujer & $-0,007 * *$ & $-0,002^{* *}$ & $-0,002^{* *}$ & $0,010^{* *}$ \\
& $(0,003)$ & $(0,001)$ & $(0,001)$ & $(0,005)$ \\
Edad & $0,004^{* * *}$ & $0,001^{* * *}$ & $0,001 * * *$ & $-0,006^{* * *}$ \\
& $(0,001)$ & $(0,000)$ & $(0,000)$ & $(0,001)$ \\
\hline Uno de los dos padres con estudios medios & $-0,002$ & $-0,001$ & $-0,001$ & 0,003 \\
& $(0,004)$ & $(0,001)$ & $(0,001)$ & $(0,007)$ \\
Los dos padres con estudios medios & $-0,008^{* *}$ & $-0,002^{* *}$ & $-0,002^{* *}$ & $0,012^{* *}$ \\
Uno de los dos padres con estudios superiores & $(0,004)$ & $(0,001)$ & $(0,001)$ & $(0,006)$ \\
& $-0,010^{*}$ & $-0,002^{* *}$ & $-0,002^{* *}$ & $0,014 * *$ \\
Los dos padres con estudios superiores & $(0,040)$ & $(0,001)$ & $(0,001)$ & $(0,006)$ \\
& $-0,019 * * *$ & $-0,005^{* * *}$ & $-0,005^{* * *}$ & $0,029 * * *$ \\
& $(0,004)$ & $(0,001)$ & $(0,001)$ & $(0,006)$ \\
\hline
\end{tabular}

FUENTE: Elaboración propia a partir de las Encuestas de Inserción Laboral (AQU).

NOTA: Los errores estándar (en paréntesis) son robustos a la heterocedasticidad. * Significativo al 10 por 100 , ** Significativo al 5 por 100 . *** Significativo al 1 por 100 . Los resultados completos correspondientes a la estimación de estos modelos se recogen en el Anexo 1. 


\section{CUADRO 4 (Continuación) \\ EFECTOS MARGINALES DEL MODELO PROBIT ORDENADO PARA EL DESAJUSTE EDUCATIVO}

\begin{tabular}{|c|c|c|c|c|}
\hline & (1) & (2) & (3) & (4) \\
\hline Nota final notable & $\begin{array}{c}-0,014 * * * \\
(0,003)\end{array}$ & $\begin{array}{c}-0,003 * * * \\
(0,001)\end{array}$ & $\begin{array}{c}-0,003 * * * \\
(0,001)\end{array}$ & $\begin{array}{c}0,020 * * * \\
(0,004)\end{array}$ \\
\hline Nota final sobresaliente & $\begin{array}{c}-0,026 * * * \\
(0,008)\end{array}$ & $\begin{array}{c}-0,006^{* * * *} \\
(0,002)\end{array}$ & $\begin{array}{c}-0,007 * * * \\
(0,002)\end{array}$ & $\begin{array}{c}0,039 * * * \\
(0,012)\end{array}$ \\
\hline Nota final matrícula de honor & $\begin{array}{c}-0,055^{* * * *} \\
(0,017)\end{array}$ & $\begin{array}{c}-0,015^{* * * *} \\
(0,005)\end{array}$ & $\begin{array}{c}-0,016^{* * * *} \\
(0,006)\end{array}$ & $\begin{array}{c}0,086 * * * \\
(0,028)\end{array}$ \\
\hline Área de ciencias sociales & $\begin{array}{c}-0,066^{* * * *} \\
(0,006)\end{array}$ & $\begin{array}{c}-0,014 * * * \\
(0,001)\end{array}$ & $\begin{array}{c}-0,015^{* * * *} \\
(0,001)\end{array}$ & $\begin{array}{c}0,095 * * * \\
(0,008)\end{array}$ \\
\hline Área de ciencias experimentales & $\begin{array}{c}-0,023 * * * \\
(0,008)\end{array}$ & $\begin{array}{c}-0,005^{* * * *} \\
(0,002)\end{array}$ & $\begin{array}{c}-0,004 * * * \\
(0,002)\end{array}$ & $\begin{array}{c}0,032 * * * \\
(0,011)\end{array}$ \\
\hline Área de ciencias de la salud & $\begin{array}{c}-0,088 * * * \\
(0,008)\end{array}$ & $\begin{array}{c}-0,020 * * * \\
(0,002)\end{array}$ & $\begin{array}{c}-0,021 * * * \\
(0,002)\end{array}$ & $\begin{array}{c}0,129 * * * \\
(0,012)\end{array}$ \\
\hline Área de disciplinas técnicas & $\begin{array}{c}-0,066^{* * *} \\
(0,008)\end{array}$ & $\begin{array}{c}-0,014 * * * \\
(0,002)\end{array}$ & $\begin{array}{c}-0,015^{* * *} \\
(0,002)\end{array}$ & $\begin{array}{c}0,095 * * * \\
(0,011)\end{array}$ \\
\hline Funciones de dirección & $\begin{array}{c}-0,042 * * * \\
(0,003)\end{array}$ & $\begin{array}{c}-0,010 * * * \\
(0,001)\end{array}$ & $\begin{array}{c}-0,011 * * * \\
(0,001)\end{array}$ & $\begin{array}{c}0,064 * * * \\
(0,005)\end{array}$ \\
\hline Funciones de comercio & $\begin{array}{c}0,043 * * * \\
(0,005)\end{array}$ & $\begin{array}{c}0,010^{* * *} * \\
(0,001)\end{array}$ & $\begin{array}{c}0,010 * * * \\
(0,001)\end{array}$ & $\begin{array}{c}-0,064 * * * \\
(0,007)\end{array}$ \\
\hline Funciones de enseñanza & $\begin{array}{c}-0,073 * * * \\
(0,005)\end{array}$ & $\begin{array}{c}-0,020 * * * \\
(0,001)\end{array}$ & $\begin{array}{c}-0,022 * * * \\
(0,002)\end{array}$ & $\begin{array}{c}0,114 * * * \\
(0,007)\end{array}$ \\
\hline Funciones de asistencia & $\begin{array}{c}-0,072 * * * \\
(0,005)\end{array}$ & $\begin{array}{c}-0,020 * * * \\
(0,002)\end{array}$ & $\begin{array}{c}-0,023 * * * \\
(0,002)\end{array}$ & $\begin{array}{c}0,115^{* * * *} \\
(0,008)\end{array}$ \\
\hline Funciones de I+D & $\begin{array}{c}-0,063 * * * \\
(0,003)\end{array}$ & $\begin{array}{c}-0,017 * * * \\
(0,001)\end{array}$ & $\begin{array}{c}-0,019 * * * \\
(0,001)\end{array}$ & $\begin{array}{c}0,100 * * * \\
(0,006)\end{array}$ \\
\hline Funciones de diseño & $\begin{array}{c}0,007 \\
(0,007)\end{array}$ & $\begin{array}{c}0,002 \\
(0,002)\end{array}$ & $\begin{array}{c}0,002 \\
(0,002)\end{array}$ & $\begin{array}{l}-0,010 \\
(0,011)\end{array}$ \\
\hline Funciones técnicas & $\begin{array}{c}-0,058^{* * *} \\
(0,003)\end{array}$ & $\begin{array}{c}-0,014 * * * \\
(0,001)\end{array}$ & $\begin{array}{c}-0,015^{* * * *} \\
(0,001)\end{array}$ & $\begin{array}{c}0,086 * * * \\
(0,005)\end{array}$ \\
\hline Otras funciones cualificadas & $\begin{array}{c}0,045 * * * \\
(0,005)\end{array}$ & $\begin{array}{c}0,010 * * * \\
(0,001)\end{array}$ & $\begin{array}{c}0,010 * * * \\
(0,001)\end{array}$ & $\begin{array}{c}-0,065 * * * \\
(0,008)\end{array}$ \\
\hline Otras funciones no cualificadas & $\begin{array}{c}0,210 * * * \\
(0,011)\end{array}$ & $\begin{array}{c}0,033 * * * \\
(0,001)\end{array}$ & $\begin{array}{c}0,029 * * * \\
(0,001)\end{array}$ & $\begin{array}{c}-0,273 * * * \\
(0,013)\end{array}$ \\
\hline
\end{tabular}

FUENTE: Elaboración propia a partir de las Encuestas de Inserción Laboral (AQU).

NOTA: Los errores estándar (en paréntesis) son robustos a la heterocedasticidad. * Significativo al 10 por 100. ** Significativo al 5 por 100. *** Significativo al 1 por 100. Los efectos marginales mostrados son promedios para toda la muestra y se corresponden a la estimación del modelo (c) del Cuadro 3 donde, además de las variables mostradas en este cuadro, se incluyen las variables ficticias correspondientes a universidad, año de la encuesta, provincia/CCAA de trabajo y características del empleo y de la empresa. 


\section{Conclusiones}

El objetivo de este trabajo ha consistido en analizar si existe una asociación entre la movilidad durante los estudios y la sobrecualificación autopercibida a partir de la información disponible en las encuestas de inserción laboral de los titulados y tituladas en las universidades catalanas.

La evidencia obtenida permite concluir que existe una relación positiva entre movilidad nacional e internacional y desajuste educativo tanto si la movilidad se ha realizado únicamente con finalidades asociadas a la realización de estudios como si se trata de la realización de prácticas u otras experiencias laborales. Una posible explicación de este resultado sería la adquisición de determinadas competencias durante la estancia en el extranjero o en otros centros universitarios españoles que permiten a estos graduados asumir algunas funciones en su puesto de trabajo en mejores condiciones, respecto a sus compañeros de promoción que no llevaron a cabo ninguna estancia de movilidad.

Sin embargo, el análisis realizado no está exento de limitaciones. En concreto, una primera limitación del estudio es que no es posible establecer una relación causal entre la movilidad internacional durante los estudios y el menor desajuste educativo, sino únicamente la existencia de una asociación entre ambas variables. Tal y como señalan Di Pietro (2014) o Waibel et al. (2018), no solo es necesario tener en cuenta el papel moderador de algunos factores observables, sino que es posible que los individuos que participan en los programas de movilidad tengan algunas características que los hagan más adecuados para realizar determinadas tareas por parte de las empresas. Sin embargo, la dificultad para tener en cuenta el diferente perfil de los participantes y poder corregir la posible autoselección de los participantes es elevada. Solo recientemente algunos estudios han intentado afrontar esta limitación a través de la realización de experimentos (Petzold, 2017a y 2017b), la aplicación de técnicas de matching (Rodrigues, 2013), métodos basados en regression discontinuity (Oosterbeek y Webbink, 2006) o estimación por variables instrumentales (Di Pietro, 2015; Messer y Wolter, 2007). Así pues, una clara extensión del trabajo consistiría en intentar aplicar estos métodos en el contexto de este estudio. En este sentido, el trabajo de Iriondo-Múgica (2017) con bases de datos similares y donde aplica Propensity Score Matching u otros estudios internacionales que adoptan una aproximación basada en variables instrumentales suponen avances significativos que podrían extenderse en el futuro al análisis realizado en este trabajo. También sería interesante analizar los resultados obtenidos con otros indicadores de desajuste educativo no subjetivos, como por ejemplo con medidas objetivas obtenidas por medio de evaluación de expertos (Leuven y Oosterbeek, 2011). Por último, otras líneas futuras consistirían en añadir la visión de los ocupadores a partir de los datos de las recientes encuestas de AQU sobre este tema, así como poder distinguir entre los distintos destinos en que se ha realizado la movilidad. Parece razonable pensar que para aquellos estudiantes donde la movilidad ha tenido un carácter «vertical», es decir, han realizado estancias o prácticas en entornos académicamente o laboralmente superiores a los de origen el 
efecto podría ser mayor, mientras que, en cambio, para aquellos estudiantes que han visitado instituciones o empresas similares a los de origen (movilidad «horizontal») podría no observarse ningún efecto.

\section{Referencias bibliográficas}

[1] AQU Catalunya (2017). 2017. La inserció laboral dels graduats i graduades de les universitats catalanes. Disponible a http://www.aqu.cat/doc/doc_56174010_1.pdf.

[2] BECKER, G. S. (1964). Human Capital: a Theoretical and Empirical Analysis, with Special Reference to Education. Nueva York: National Bureau of Economic Research.

[3] BÜCHEL, F.; VAN HAM, M. (2003). «Overeducation, Regional Labour Markets and Spatial Flexibility». Journal of Urban Economics, 53 (3), 482-493.

[4] CRESCENZI, R.; GAGLIARDI, L. y ORRU', E. (2016). «Learning mobility grants and skill (mis)matching in the labour market: The case of the 'Master and Back' Programme». Papers in Regional Science, 95, 693-707.

[5] DAVIA, M. A.; McGUINNESS, S. y O'CONNELL, P. J. (2017). «Determinants of regional differences in rates of overeducation in Europe", Social Science Research, 63, 67-80.

[6] DI PIETRO, G. (2012). «Does studying abroad cause international labor mobility? Evidence from Italy». Economics Letters, 117 (3), 632-635.

[7] DI PIETRO, G. (2014). «University study abroad and graduates' employability». IZA World of Labor, 109 (doi: 10.15185/izawol.109).

[8] DI PIETRO, G. (2015). «Do study abroad programs enhance the employability of graduates?». Education, Finance and Policy, 10 (2), 223-243.

[9] EUROPEAN COMMISSION (2014). The Erasmus Impact Study. Effects of mobility on the skills and employability of students and the internationalisation of higher education institutions. Luxemburgo: Publications Office of the European Union.

[10] FRIEDBERG, R. M. (2000). «You can't take it with you? Immigrant assimilation and the portability of human capital». Journal of Labour Economics, 18 (2), 221-251.

[11] HENSEN, M. M.; DE VRIES, M. R. y CÖRVERS, F. (2009). «The role of geographic mobility in reducing education-job mismatches in the Netherlands». Papers in Regional Science, 88 (3), 667-682.

[12] IAMMARINO, S. y MARINELLI, E. (2015). «Education - Job (Mis)Match and Interregional Migration: Italian University Graduates' Transition to Work». Regional Studies, 49 (5), 866-882.

[13] IRIONDO MÚGICA, I. (2017). «Evaluación del impacto de la movilidad Erasmus en los salarios y el empleo de los recién titulados en España». Documentos de Trabajo de la Facultad de Ciencias Económicas y Empresariales de la Universidad Complutense de Madrid, 17-04.

[14] KAZAKIS, P. y FAGGIAN, A. (2017). «Mobility, education and labor market outcomes for U.S. graduates: Is selectivity important?». The Annals of Regional Science, 59 (3), 731-758.

[15] LEUVEN, E. y OOSTERBEEK, H. (2011). «Overeducation and mismatch in the labor market», en Handbook of the Economics of Education, 4, 283-326. Elsevier. 
[16] McGUINNESS, S.; POULIAKAS, K. y REDMOND, P. (2018). «Skills mismatch: concepts, measurement and policy approaches, Journal of Economic Surveys, 32, 9851015.

[17] MESSER, D. y WOLTER, S. C. (2007). «Are student exchange programs worth it?». High Education, 54, pp. 647-663.

[18] MURILLO, I. P. y RAYMOND, J. Ll. (2017). «Es rentable invertir en educación? Los rendimientos de la inversión educativa España y Catalunya en el periodo 2002-2010». Revista de Economía, 13, 36-45.

[19] OOSTERBEEK, H. y WEBBINK, D. (2006). «Assessing the returns to studying abroad». CPB Discussion Paper 64.

[20] PAREY, M. y WALDINGER, F. (2011). «Studying abroad and the effect of international labour market mobility: Evidence from the introduction of Erasmus». Economic Journal, 121 (551), 194-222.

[21] PETZOLD, K. (2017a). «Studying abroad as a sorting criterion in the recruitment process. A field experiment among German employers». Journal of Studies in International Education, 21 (5), 412-430.

[22] PETZOLD, K. (2017b). «The role of international student mobility in hiring decisions. A vignette experiment among German employers». Journal of Education and Work, 30 (8), 893-911.

[23] RAMOS, R. y SANROMÁ, E. (2013). «Overeducation and local labour markets in Spain». Tijdschrift Voor Economische en Sociale Geografie, 104 (3), 278-291.

[24] RODRIGUES, M. (2013). Does Student Mobility during Higher Education Pay? Evidence from 16 European Countries. Ispra, Italia: Joint Research CENTRE, European Commission.

[25] ROSPIGLIOSI, A. P.; GREENER, S.; BOURNER, T. y SHEEHAN, M. (2014). «Human capital or signalling, unpacking the graduate premium». International Journal of Social Economics, 41 (5), 420-432.

[26] SANROMA,E.; RAMOS, R. y SIMÓN,H.(2015). «How relevant is the origin of human capital for immigrant wages? Evidence from Spain». Journal of Applied Economics, 18 (1), 149-172.

[27] SPENCE, M. (1973). «Job Market Signaling». Quarterly Journal of Economics, 87, 355-374.

[28] TEICHLER, U. y JANSON, K. (2007). «The professional value of temporary study in another European country: Employment and work of former Erasmus students». Journal of Studies in International Education, 11 (3-4), 486-495.

[29] VAN MOL, C. (2017). «Do employers value international study and internships? A comparative analysis of 31 countries». Geoforum, 78, 52-60.

[30] WAIBEL, S.; RÜGER, H.; ETTE, A. y Sauer, L. (2017). «Career consequences of transnational educational mobility: A systematic literature review». Educational Research Review, 20, 81-98.

[31] WAIBEL, S.; PETZOLD, K. y RÜGER, H. (2018). «Occupational status benefits of studying abroad and the role of occupational specificity - A propensity score matching approach». Social Science Research, 74, 45-61. 
ANEXO 1

ESTADÍSTICAS DESCRIPTIVAS DE LAS VARIABLES ANALIZADAS

\begin{tabular}{|c|c|c|c|c|c|c|c|c|c|c|}
\hline & \multicolumn{2}{|c|}{ Todos } & \multicolumn{2}{|c|}{2008} & \multicolumn{2}{|c|}{2011} & \multicolumn{2}{|c|}{2014} & \multicolumn{2}{|c|}{2017} \\
\hline & Media & $D T$ & Media & $D T$ & Media & $D T$ & Media & $D T$ & Media & $D T$ \\
\hline $\begin{array}{l}\text { Ningún requisito, fun- } \\
\text { ciones no universita- } \\
\text { rias }\end{array}$ & 0,115 & 0,319 & 0,094 & 0,292 & 0,088 & 0,283 & 0,157 & 0,364 & 0,128 & 0,334 \\
\hline $\begin{array}{l}\text { Requisito de titulación } \\
\text { universitaria, funcio- } \\
\text { nes no propias }\end{array}$ & 0,037 & 0,189 & 0,040 & 0,196 & 0,040 & 0,195 & 0,044 & 0,204 & 0,027 & 0,162 \\
\hline $\begin{array}{l}\text { Requisito de titula- } \\
\text { ción específica, fun- } \\
\text { ciones no propias de } \\
\text { la titulación especí- } \\
\text { fica }\end{array}$ & 0,048 & 0,214 & 0,054 & 0,227 & 0,054 & 0,225 & 0,027 & 0,163 & 0,053 & 0,223 \\
\hline $\begin{array}{l}\text { Funciones propias de } \\
\text { la titulación especí- } \\
\text { fica (independiente- } \\
\text { mente del requisito) }\end{array}$ & 0,800 & 0,400 & 0,812 & 0,391 & 0,819 & 0,385 & 0,772 & 0,419 & 0,792 & 0,406 \\
\hline No movilidad & 0,630 & 0,483 & 0,680 & 0,466 & 0,612 & 0,487 & 0,595 & 0,491 & 0,622 & 0,485 \\
\hline Movilidad académica & 0,175 & 0,380 & 0,137 & 0,344 & 0,170 & 0,376 & 0,214 & 0,410 & 0,190 & 0,393 \\
\hline Movilidad laboral & 0,111 & 0,314 & 0,119 & 0,324 & 0,122 & 0,327 & 0,108 & 0,310 & 0,096 & 0,295 \\
\hline $\begin{array}{l}\text { Movilidad académi- } \\
\text { ca y laboral }\end{array}$ & 0,083 & 0,276 & 0,064 & 0,245 & 0,096 & 0,295 & 0,083 & 0,276 & 0,091 & 0,288 \\
\hline Mujer & 0,606 & 0,489 & 0,616 & 0,486 & 0,616 & 0,486 & 0,605 & 0,489 & 0,587 & 0,492 \\
\hline Edad & 29,00 & 3,740 & 28,80 & 3,293 & 29,13 & 3,706 & 29,10 & 3,993 & 29,00 & 3,990 \\
\hline $\begin{array}{l}\text { Padres con estudios } \\
\text { primarios o sin estu- } \\
\text { dios }\end{array}$ & 0,337 & 0,473 & 0,403 & 0,491 & 0,362 & 0,481 & 0,320 & 0,467 & 0,254 & 0,435 \\
\hline $\begin{array}{l}\text { Uno de los dos padres } \\
\text { con estudios medios }\end{array}$ & 0,127 & 0,333 & 0,153 & 0,360 & 0,127 & 0,333 & 0,101 & 0,302 & 0,121 & 0,327 \\
\hline $\begin{array}{l}\text { Los dos padres con } \\
\text { estudios medios }\end{array}$ & 0,182 & 0,386 & 0,159 & 0,365 & 0,184 & 0,388 & 0,198 & 0,398 & 0,191 & 0,393 \\
\hline $\begin{array}{l}\text { Uno de los dos pa- } \\
\text { dres con estudios su- } \\
\text { periores }\end{array}$ & 0,184 & 0,387 & 0,167 & 0,373 & 0,172 & 0,377 & 0,185 & 0,388 & 0,213 & 0,409 \\
\hline $\begin{array}{l}\text { Los dos padres con } \\
\text { estudios superiores }\end{array}$ & 0,170 & 0,376 & 0,118 & 0,323 & 0,154 & 0,361 & 0,196 & 0,397 & 0,221 & 0,415 \\
\hline Nota final aprobado & 0,421 & 0,494 & 0,458 & 0,498 & 0,481 & 0,500 & 0,438 & 0,496 & 0,310 & 0,463 \\
\hline Nota final notable & 0,546 & 0,498 & 0,504 & 0,500 & 0,489 & 0,500 & 0,534 & 0,499 & 0,653 & 0,476 \\
\hline $\begin{array}{l}\text { Nota final sobresa- } \\
\text { liente }\end{array}$ & 0,028 & 0,165 & 0,033 & 0,179 & 0,024 & 0,154 & 0,023 & 0,151 & 0,030 & 0,171 \\
\hline $\begin{array}{l}\text { Nota final matrícula } \\
\text { de honor }\end{array}$ & 0,005 & 0,073 & 0,005 & 0,069 & 0,006 & 0,076 & 0,004 & 0,064 & 0,006 & 0,079 \\
\hline
\end{tabular}

FUENTE: Elaboración propia a partir de las Encuestas de Inserción Laboral (AQU).

NOTA: DT corresponde a desviación típica. 
ANEXO 1 (Continuación)

ESTADÍSTICAS DESCRIPTIVAS DE LAS VARIABLES ANALIZADAS

\begin{tabular}{|c|c|c|c|c|c|c|c|c|c|c|}
\hline & \multicolumn{2}{|c|}{ Todos } & \multicolumn{2}{|c|}{2008} & \multicolumn{2}{|c|}{2011} & \multicolumn{2}{|c|}{2014} & \multicolumn{2}{|c|}{2017} \\
\hline & Media & $D T$ & Media & $D T$ & Media & $D T$ & Media & $D T$ & Media & $D T$ \\
\hline Área de humanidades & 0,100 & 0,300 & 0,112 & 0,315 & 0,099 & 0,299 & 0,085 & 0,279 & 0,100 & 0,301 \\
\hline $\begin{array}{l}\text { Área de ciencias so- } \\
\text { ciales }\end{array}$ & 0,463 & 0,499 & 0,464 & 0,499 & 0,455 & 0,498 & 0,481 & 0,500 & 0,457 & 0,498 \\
\hline $\begin{array}{l}\text { Área de ciencias ex- } \\
\text { perimentales }\end{array}$ & 0,082 & 0,274 & 0,083 & 0,276 & 0,079 & 0,270 & 0,080 & 0,272 & 0,084 & 0,277 \\
\hline $\begin{array}{l}\text { Área de ciencias de } \\
\text { la salud }\end{array}$ & 0,113 & 0,317 & 0,098 & 0,298 & 0,102 & 0,302 & 0,113 & 0,316 & 0,141 & 0,348 \\
\hline $\begin{array}{l}\text { Área de disciplinas } \\
\text { técnicas }\end{array}$ & 0,242 & 0,428 & 0,243 & 0,429 & 0,265 & 0,442 & 0,241 & 0,428 & 0,219 & 0,413 \\
\hline UB & & 0,448 & ,267 & 0,442 & 0,255 & 0,436 & 0,271 & 0,444 & 0,318 &, 466 \\
\hline UAB & 0,220 & 0,414 & 0,213 & 0,409 & 0,226 & 0,418 & 0,208 & 0,406 & 0,232 & 0,422 \\
\hline UPC & 0,151 & 0,358 & 0,146 & 0,353 & 0,159 & 0,365 & 0,151 & 0,358 & 0,150 & 0,357 \\
\hline UPF & 0,081 & 0,273 & 0,082 & 0,274 & 0,073 & 0,260 & 0,091 & 0,288 & 0,079 & 0,270 \\
\hline UdG & 0,089 & 0,285 & 0,098 & 0,297 & 0,092 & 0,289 & 0,091 & 0,288 & 0,075 & 0,264 \\
\hline UdL & 0,070 & 0,255 & 0,084 & 0,277 & 0,070 & 0,256 & 0,062 & 0,242 & 0,062 & 0,241 \\
\hline URV & 0,111 & 0,314 & 0,111 & 0,314 & 0,126 & 0,332 & 0,126 & 0,332 & 0,083 & 0,276 \\
\hline Trabaja en Barcelona & 0,677 & 0,468 & 0,658 & 0,474 & 0,661 & 0,473 & 0,659 & 0,474 & 0,726 & 0,446 \\
\hline Trabaja en Girona & 0,102 & 0,303 & 0,110 & 0,312 & 0,114 & 0,318 & 0,111 & 0,314 & 0,076 & 0,264 \\
\hline Trabaja en Lleida & 0,092 & 0,289 & 0,103 & 0,304 & 0,098 & 0,297 & 0,092 & 0,289 & 0,075 & 0,264 \\
\hline Trabaja en Tarragona & 0,060 & 0,237 & 0,069 & 0,254 & 0,060 & 0,238 & 0,059 & 0,236 & 0,050 & 0,218 \\
\hline $\begin{array}{l}\text { Trabaja en otras CC. } \\
\text { AA. españolas }\end{array}$ & 0,069 & 0,254 & 0,060 & 0,238 & 0,067 & 0,250 & 0,079 & 0,269 & 0,074 & 0,261 \\
\hline Sector privado & & & & 0,471 & 0,672 & 0,469 & 0,824 & 0,380 & 0,786 & 0,410 \\
\hline $\begin{array}{l}\text { Jornada a tiempo } \\
\text { completo }\end{array}$ & 0,805 & 0,396 & 0,116 & 0,320 & 0,161 & 0,367 & 0,725 & 0,447 & 0,246 & 0,431 \\
\hline $\begin{array}{l}\text { Contracto fijo/indefi- } \\
\text { nido }\end{array}$ & 0,540 & 0,498 & 0,593 & 0,491 & 0,577 & 0,494 & 0,489 & 0,500 & 0,488 & 0,500 \\
\hline Trabajador autónomo & 0,091 & 0,288 & 0,077 & 0,266 & 0,073 & 0,260 & 0,117 & 0,321 & 0,103 & 0,304 \\
\hline Contrato temporal & 0,363 & 0,481 & 0,326 & 0,469 & 0,344 & 0,475 & 0,384 & 0,486 & 0,403 & 0,490 \\
\hline $\begin{array}{l}\text { Becarios u otras si- } \\
\text { tuaciones }\end{array}$ & 0,007 & 0,082 & 0,005 & 0,067 & 0,007 & 0,082 & 0,010 & 0,101 & 0,006 & 0,078 \\
\hline Antigüedad laboral & 2,357 & 1,276 & 2,320 & 1,226 & 2,761 & 1,356 & 2,339 & 1,258 & 2,029 & 1,157 \\
\hline
\end{tabular}

FUENTE: Elaboración propia a partir de las Encuestas de Inserción Laboral (AQU).

NOTA: DT corresponde a desviación típica. 
ANEXO 1 (Continuación)

ESTADÍSTICAS DESCRIPTIVAS DE LAS VARIABLES ANALIZADAS

\begin{tabular}{|c|c|c|c|c|c|c|c|c|c|c|}
\hline & \multicolumn{2}{|c|}{ Todos } & \multicolumn{2}{|c|}{2008} & \multicolumn{2}{|c|}{2011} & \multicolumn{2}{|c|}{2014} & \multicolumn{2}{|c|}{2017} \\
\hline & Media & $D T$ & Media & $D T$ & Media & $D T$ & Media & $D T$ & Media & $D T$ \\
\hline $\begin{array}{l}\text { Agricultura, ganade- } \\
\text { ría, silvicultura, caza }\end{array}$ & 0,012 & 0,108 & 0,011 & 0,106 & 0,005 & 0,072 & 0,016 & 0,127 & 0,015 & 0,121 \\
\hline $\begin{array}{l}\text { Pesca y piscicultura, } \\
\text { acuicultura de aguas } \\
\text { continentales o ma- } \\
\text { rinas }\end{array}$ & 0,000 & 0,019 & 0,000 & 0,015 & 0,000 & 0,012 & 0,000 & 0,022 & 0,001 & 0,025 \\
\hline $\begin{array}{l}\text { Comb. sólidos, petró- } \\
\text { leo, gas y minerales } \\
\text { radiactivos }\end{array}$ & 0,002 & 0,049 & 0,002 & 0,045 & 0,001 & 0,036 & 0,004 & 0,065 & 0,002 & 0,049 \\
\hline $\begin{array}{l}\text { Electricidad, gas y } \\
\text { agua. Fabricación de } \\
\text { generadores de vapor, } \\
\text { captación, depuración } \\
\text { y distrib. de agua }\end{array}$ & 0,014 & 0,119 & 0,013 & 0,113 & 0,017 & 0,128 & 0,014 & 0,118 & 0,013 & 0,115 \\
\hline $\begin{array}{l}\text { Extracción y transfor- } \\
\text { mación de minerales }\end{array}$ & 0,001 & 0,030 & 0,001 & 0,031 & 0,001 & 0,028 & 0,001 & 0,035 & 0,001 & 0,025 \\
\hline Industrias químicas & 0,015 & 0,122 & 0,015 & 0,121 & 0,011 & 0,103 & 0,015 & 0,121 & 0,019 & 0,137 \\
\hline $\begin{array}{l}\text { Industrias farmacéuti- } \\
\text { cas y cosméticas }\end{array}$ & 0,024 & 0,154 & 0,018 & 0,132 & 0,017 & 0,129 & 0,027 & 0,162 & 0,037 & 0,188 \\
\hline $\begin{array}{l}\text { Metalurgia, material } \\
\text { eléctrico y de preci- } \\
\text { sión }\end{array}$ & 0,030 & 0,170 & 0,033 & 0,179 & 0,025 & 0,157 & 0,030 & 0,172 & 0,030 & 0,172 \\
\hline $\begin{array}{l}\text { Materiales de trans- } \\
\text { porte. Fabricación ve- } \\
\text { hículos motor, bici- } \\
\text { cletas, construcción } \\
\text { naval, ... }\end{array}$ & 0,015 & 0,121 & 0,012 & 0,109 & 0,011 & 0,106 & 0,021 & 0,142 & 0,017 & 0,129 \\
\hline $\begin{array}{l}\text { Productos alimenta- } \\
\text { rios, bebidas y tabaco }\end{array}$ & 0,025 & 0,157 & 0,021 & 0,144 & 0,023 & 0,149 & 0,026 & 0,159 & 0,031 & 0,174 \\
\hline $\begin{array}{l}\text { Industrias textiles, del } \\
\text { cuero y de confeccio- } \\
\text { nes }\end{array}$ & 0,009 & 0,095 & 0,006 & 0,077 & 0,005 & 0,074 & 0,011 & 0,103 & 0,014 & 0,119 \\
\hline $\begin{array}{l}\text { Industrias de la made- } \\
\text { ra, corcho y muebles } \\
\text { de madera }\end{array}$ & 0,001 & 0,034 & 0,002 & 0,039 & 0,001 & 0,026 & 0,001 & 0,035 & 0,001 & 0,034 \\
\hline $\begin{array}{l}\text { Papel y artículos deri- } \\
\text { vados. Artes gráficas } \\
\text { y edición. Fabrica- } \\
\text { ción de pasta de pa- } \\
\text { pel, cartón }\end{array}$ & 0,007 & 0,081 & 0,010 & 0,100 & 0,004 & 0,061 & 0,004 & 0,066 & 0,008 & 0,087 \\
\hline $\begin{array}{l}\text { Caucho y plástico. } \\
\text { Otras industrias ma- } \\
\text { nufactureras. Recicla- } \\
\text { je. Fabricación de vi- } \\
\text { drio, fibras sintéticas } \\
\text { Construcción }\end{array}$ & 0,003 & 0,056 & 0,003 & 0,059 & 0,003 & 0,054 & 0,003 & 0,056 & 0,003 & 0,054 \\
\hline
\end{tabular}

FUENTE: Elaboración propia a partir de las Encuestas de Inserción Laboral (AQU). NOTA: DT corresponde a desviación típica. 
ANEXO 1 (Continuación)

ESTADÍSTICAS DESCRIPTIVAS DE LAS VARIABLES ANALIZADAS

\begin{tabular}{|c|c|c|c|c|c|c|c|c|c|c|}
\hline & \multicolumn{2}{|c|}{ Todos } & \multicolumn{2}{|c|}{2008} & \multicolumn{2}{|c|}{2011} & \multicolumn{2}{|c|}{2014} & \multicolumn{2}{|c|}{2017} \\
\hline & Media & $D T$ & Media & $D T$ & Media & $D T$ & Media & $D T$ & Media & $D T$ \\
\hline $\begin{array}{l}\text { Comercio y repara- } \\
\text { ciones }\end{array}$ & 0,042 & 0,200 & 0,036 & 0,186 & 0,039 & 0,195 & 0,062 & 0,242 & 0,034 & 0,182 \\
\hline $\begin{array}{l}\text { Restaurantes, cafés y } \\
\text { hostelería }\end{array}$ & 0,015 & 0,121 & 0,007 & 0,085 & 0,010 & 0,100 & 0,022 & 0,147 & 0,022 & 0,146 \\
\hline $\begin{array}{l}\text { Transporte y activi- } \\
\text { dades afines }\end{array}$ & 0,028 & 0,166 & 0,019 & 0,138 & 0,022 & 0,148 & 0,028 & 0,166 & 0,043 & 0,203 \\
\hline $\begin{array}{l}\text { Tecnologías de co- } \\
\text { municación }\end{array}$ & 0,051 & 0,220 & 0,042 & 0,200 & 0,056 & 0,231 & 0,063 & 0,243 & 0,047 & 0,211 \\
\hline $\begin{array}{l}\text { Medios de comuni- } \\
\text { cación (radio, tele- } \\
\text { visión, cine, vídeo, } \\
\text { editoriales...) }\end{array}$ & 0,024 & 0,153 & 0,023 & 0,151 & 0,025 & 0,157 & 0,019 & 0,136 & 0,028 & 0,164 \\
\hline $\begin{array}{l}\text { Instituciones finan- } \\
\text { cieras, seguros y acti- } \\
\text { vidades inmobiliarias }\end{array}$ & 0,058 & 0,235 & 0,080 & 0,271 & 0,057 & 0,232 & 0,038 & 0,192 & 0,053 & 0,224 \\
\hline $\begin{array}{l}\text { Servicios a las em- } \\
\text { presas. Alquiler de } \\
\text { bienes }\end{array}$ & 0,122 & 0,327 & 0,113 & 0,316 & 0,117 & 0,322 & 0,145 & 0,352 & 0,118 & 0,322 \\
\hline $\begin{array}{l}\text { Administración pú- } \\
\text { blica, defensa, y Se- } \\
\text { guridad Social }\end{array}$ & 0,052 & 0,222 & 0,081 & 0,273 & 0,058 & 0,234 & 0,028 & 0,166 & 0,033 & 0,179 \\
\hline $\begin{array}{l}\text { Educación, investi- } \\
\text { gación y servicios } \\
\text { culturales }\end{array}$ & 0,252 & 0,434 & 0,254 & 0,435 & 0,298 & 0,457 & 0,232 & 0,422 & 0,222 & 0,416 \\
\hline $\begin{array}{l}\text { Sanidad y asistencia } \\
\text { social }\end{array}$ & 0,119 & 0,324 & 0,094 & 0,292 & 0,126 & 0,332 & 0,136 & 0,343 & 0,126 & 0,332 \\
\hline $\begin{array}{l}\text { Otros servicios pres- } \\
\text { tados a la comunidad }\end{array}$ & 0,030 & 0,169 & 0,036 & 0,185 & 0,019 & 0,135 & 0,024 & 0,153 & 0,038 & 0,191 \\
\hline $\begin{array}{l}\text { Empresa de hasta } 10 \\
\text { trabajadores }\end{array}$ & 0,197 & 0,398 & 0,180 & 0,385 & 0,168 & 0,374 & 0,236 & 0,425 & 0,212 & 0,409 \\
\hline $\begin{array}{l}\text { Empresa de } 11-50 \\
\text { trabajadores }\end{array}$ & 0,261 & 0,439 & 0,295 & 0,456 & 0,266 & 0,442 & 0,246 & 0,431 & 0,232 & 0,422 \\
\hline $\begin{array}{l}\text { Empresa de } 51-100 \\
\text { trabajadores }\end{array}$ & 0,106 & 0,308 & 0,114 & 0,317 & 0,100 & 0,300 & 0,113 & 0,316 & 0,100 & 0,300 \\
\hline $\begin{array}{l}\text { Empresa de } 101-250 \\
\text { trabajadores }\end{array}$ & 0,089 & 0,284 & 0,090 & 0,287 & 0,086 & 0,280 & 0,089 & 0,285 & 0,090 & 0,286 \\
\hline $\begin{array}{l}\text { Empresa de } 251-500 \\
\text { trabajadores }\end{array}$ & 0,060 & 0,237 & 0,063 & 0,242 & 0,058 & 0,234 & 0,054 & 0,226 & 0,064 & 0,245 \\
\hline $\begin{array}{l}\text { Empresa de más de } \\
500 \text { trabajadores }\end{array}$ & 0,261 & 0,439 & 0,254 & 0,435 & 0,304 & 0,460 & 0,254 & 0,435 & 0,233 & 0,423 \\
\hline $\begin{array}{l}\text { No información so- } \\
\text { bre el tamaño de la } \\
\text { empresa }\end{array}$ & 0,025 & 0,158 & 0,005 & 0,067 & 0,018 & 0,135 & 0,008 & 0,088 & 0,069 & 0,253 \\
\hline
\end{tabular}

FUENTE: Elaboración propia a partir de las Encuestas de Inserción Laboral (AQU).

NOTA: DT corresponde a desviación típica. 
ANEXO 1 (Continuación)

ESTADÍSTICAS DESCRIPTIVAS DE LAS VARIABLES ANALIZADAS

\begin{tabular}{|c|c|c|c|c|c|c|c|c|c|c|}
\hline & \multicolumn{2}{|c|}{ Todos } & \multicolumn{2}{|c|}{2008} & \multicolumn{2}{|c|}{2011} & \multicolumn{2}{|c|}{2014} & \multicolumn{2}{|c|}{2017} \\
\hline & Media & $D T$ & Media & $D T$ & Media & $D T$ & Media & $D T$ & Media & $D T$ \\
\hline $\begin{array}{l}\text { Funciones de direc- } \\
\text { ción }\end{array}$ & 0,283 & 0,450 & 0,328 & 0,470 & 0,272 & 0,445 & 0,270 & 0,444 & 0,255 & 0,436 \\
\hline $\begin{array}{l}\text { Funciones de comer- } \\
\text { cio }\end{array}$ & 0,139 & 0,345 & 0,160 & 0,366 & 0,129 & 0,335 & 0,143 & 0,350 & 0,121 & 0,327 \\
\hline $\begin{array}{l}\text { Funciones de ense- } \\
\text { ñanza }\end{array}$ & 0,249 & 0,432 & 0,272 & 0,445 & 0,298 & 0,457 & 0,206 & 0,405 & 0,211 & 0,408 \\
\hline $\begin{array}{l}\text { Funciones de asis- } \\
\text { tencia }\end{array}$ & 0,103 & 0,304 & 0,088 & 0,283 & 0,109 & 0,312 & 0,110 & 0,313 & 0,109 & 0,311 \\
\hline Funciones de I+D & 0,120 & 0,325 & 0,144 & 0,351 & 0,136 & 0,343 & 0,076 & 0,265 & 0,114 & 0,318 \\
\hline Funciones de diseño & 0,046 & 0,209 & 0,050 & 0,217 & 0,047 & 0,212 & 0,043 & 0,202 & 0,042 & 0,201 \\
\hline Funciones técnicas & 0,414 & 0,493 & 0,405 & 0,491 & 0,445 & 0,497 & 0,427 & 0,495 & 0,385 & 0,487 \\
\hline $\begin{array}{l}\text { Otras funciones cua- } \\
\text { lificadas }\end{array}$ & 0,119 & 0,324 & 0,320 & 0,466 & 0,028 & 0,165 & 0,049 & 0,215 & 0,049 & 0,215 \\
\hline $\begin{array}{l}\text { Otras funciones no } \\
\text { cualificadas }\end{array}$ & 0,045 & 0,208 & 0,097 & 0,296 & 0,017 & 0,130 & 0,032 & 0,175 & 0,027 & 0,163 \\
\hline $\begin{array}{l}\text { Número de observa- } \\
\text { ciones }\end{array}$ & 30.265 & & 8.443 & & 7.516 & & 6.368 & & 7.938 & \\
\hline
\end{tabular}

FUENTE: Elaboración propia a partir de las Encuestas de Inserción Laboral (AQU).

NOTA: DT corresponde a desviación típica. 
ANEXO 2

ESTIMACIÓN DE LOS MODELOS PROBIT ORDENADO PARA EL DESAJUSTE EDUCATIVO

\begin{tabular}{|c|c|c|c|}
\hline & (a) & (b) & (c) \\
\hline No movilidad & \multicolumn{3}{|c|}{ Categoría de referencia } \\
\hline Movilidad académica & $\begin{array}{c}0,026 \\
(0,023)\end{array}$ & $\begin{array}{c}0,070 * * * \\
(0,024)\end{array}$ & $\begin{array}{c}0,054 * * \\
(0,024)\end{array}$ \\
\hline Movilidad laboral & $\begin{array}{c}0,041 \\
(0,027)\end{array}$ & $\begin{array}{c}0,089 * * * \\
(0,028)\end{array}$ & $\begin{array}{c}0,058 * * \\
(0,029)\end{array}$ \\
\hline Movilidad académica y laboral & $\begin{array}{c}0,045 \\
(0,030)\end{array}$ & $\begin{array}{c}0,105 * * * \\
(0,032)\end{array}$ & $\begin{array}{c}0,076^{* *} \\
(0,033)\end{array}$ \\
\hline Mujer & $\begin{array}{c}0,040 * * \\
(0,018)\end{array}$ & $\begin{array}{c}0,017 \\
(0,019)\end{array}$ & $\begin{array}{c}0,043 * * \\
(0,020)\end{array}$ \\
\hline Edad & $\begin{array}{c}-0,095^{* * *} \\
(0,019)\end{array}$ & $\begin{array}{c}-0,096 * * * \\
(0,020)\end{array}$ & $\begin{array}{c}-0,102 * * * \\
(0,020)\end{array}$ \\
\hline Edad2 & $\begin{array}{c}0,001 * * * \\
(0,000)\end{array}$ & $\begin{array}{c}0,001 * * * \\
(0,000)\end{array}$ & $\begin{array}{c}0,001 * * * * \\
(0,000)\end{array}$ \\
\hline Padres con estudios primarios o sin estudios & \multicolumn{3}{|c|}{ Categoría de referencia } \\
\hline Uno de los dos padres con estudios medios & $\begin{array}{c}-0,003 \\
(0,026)\end{array}$ & $\begin{array}{c}0,009 \\
(0,027)\end{array}$ & $\begin{array}{c}0,013 \\
(0,028)\end{array}$ \\
\hline Los dos padres con estudios medios & $\begin{array}{c}0,038 \\
(0,024)\end{array}$ & $\begin{array}{c}0,049 * * \\
(0,025)\end{array}$ & $\begin{array}{c}0,052^{* *} \\
(0,025)\end{array}$ \\
\hline Uno de los dos padres con estudios superiores & $\begin{array}{c}0,058^{* *} \\
(0,024)\end{array}$ & $\begin{array}{c}0,073 * * * \\
(0,025)\end{array}$ & $\begin{array}{c}0,062 * * \\
(0,025)\end{array}$ \\
\hline Los dos padres con estudios superiores & $\begin{array}{c}0,159 * * * \\
(0,025)\end{array}$ & $\begin{array}{c}0,153 * * * \\
(0,026)\end{array}$ & $\begin{array}{c}0,128 * * * \\
(0,027)\end{array}$ \\
\hline Nota final aprobado & \multicolumn{3}{|c|}{ Categoría de referencia } \\
\hline Nota final notable & $\begin{array}{c}0,187 * * * \\
(0,017)\end{array}$ & $\begin{array}{c}0,129 * * * \\
(0,018)\end{array}$ & $\begin{array}{c}0,088 * * * \\
(0,019)\end{array}$ \\
\hline Nota final sobresaliente & $\begin{array}{c}0,381 * * * \\
(0,055)\end{array}$ & $\begin{array}{c}0,251 * * * \\
(0,057)\end{array}$ & $\begin{array}{c}0,172 * * * \\
(0,058)\end{array}$ \\
\hline Nota final matrícula de honor & $\begin{array}{c}0,647 * * * \\
(0,143)\end{array}$ & $\begin{array}{c}0,489 * * * \\
(0,152)\end{array}$ & $\begin{array}{c}0,423 * * \\
(0,164)\end{array}$ \\
\hline Área de humanidades & \multicolumn{3}{|c|}{ Categoría de referencia } \\
\hline Área de ciencias sociales & $\begin{array}{c}0,492 * * * \\
(0,027)\end{array}$ & $\begin{array}{c}0,440 * * * \\
(0,029)\end{array}$ & $\begin{array}{c}0,370 * * * \\
(0,030)\end{array}$ \\
\hline Área de ciencias experimentales & $\begin{array}{c}0,387 * * * \\
(0,035)\end{array}$ & $\begin{array}{c}0,300 * * * \\
(0,038)\end{array}$ & $\begin{array}{c}0,116^{* * * *} \\
(0,040)\end{array}$ \\
\hline Área de ciencias de la salud & $\begin{array}{c}0,828 * * * \\
(0,037)\end{array}$ & $\begin{array}{c}0,725 * * * \\
(0,047)\end{array}$ & $\begin{array}{c}0,532 * * * \\
(0,051)\end{array}$ \\
\hline Área de disciplinas técnicas & $\begin{array}{c}0,603 * * * \\
(0,036)\end{array}$ & $\begin{array}{c}0,634 * * * \\
(0,041)\end{array}$ & $\begin{array}{c}0,370 * * * \\
(0,043)\end{array}$ \\
\hline
\end{tabular}

FUENTE: Elaboración propia a partir de las Encuestas de Inserción Laboral (AQU).

NOTA: Los errores estándar (en paréntesis) son robustos a la heterocedasticidad. * Significativo al 10 por 100 , ** Significativo al 5 por 100 . *** Significativo al 1 por 100. 
ANEXO 2 (Continuación)

ESTIMACIÓN DE LOS MODELOS PROBIT ORDENADO PARA EL DESAJUSTE EDUCATIVO

\begin{tabular}{|c|c|c|c|}
\hline & (a) & (b) & (c) \\
\hline UB & \multicolumn{3}{|c|}{ Categoría de referencia } \\
\hline UAB & $\begin{array}{c}-0,039^{*} \\
(0,023)\end{array}$ & $\begin{array}{c}-0,049 * * \\
(0,024)\end{array}$ & $\begin{array}{c}-0,034 \\
(0,025)\end{array}$ \\
\hline UPC & $\begin{array}{c}0,081^{* *} \\
(0,036)\end{array}$ & $\begin{array}{c}0,037 \\
(0,038)\end{array}$ & $\begin{array}{c}0,035 \\
(0,039)\end{array}$ \\
\hline UPF & $\begin{array}{c}0,101 * * * \\
(0,034)\end{array}$ & $\begin{array}{c}0,157 * * * \\
(0,036)\end{array}$ & $\begin{array}{c}0,161 * * * \\
(0,037)\end{array}$ \\
\hline UdG & $\begin{array}{l}-0,037 \\
(0,032)\end{array}$ & $\begin{array}{l}-0,056 \\
(0,044)\end{array}$ & $\begin{array}{l}-0,029 \\
(0,045)\end{array}$ \\
\hline UdL & $\begin{array}{c}0,027 \\
(0,036)\end{array}$ & $\begin{array}{c}0,009 \\
(0,049)\end{array}$ & $\begin{array}{c}0,005 \\
(0,051)\end{array}$ \\
\hline URV & $\begin{array}{c}0,004 \\
(0,030)\end{array}$ & $\begin{array}{c}0,034 \\
(0,040)\end{array}$ & $\begin{array}{c}0,026 \\
(0,042)\end{array}$ \\
\hline Año 2008 & \multicolumn{3}{|c|}{ Categoría de referencia } \\
\hline Año 2011 & $\begin{array}{c}0,014 \\
(0,023)\end{array}$ & $\begin{array}{l}-0,014 \\
(0,024)\end{array}$ & $\begin{array}{c}-0,203 * * * \\
(0,027)\end{array}$ \\
\hline Año 2014 & $\begin{array}{c}-0,236 * * * \\
(0,024)\end{array}$ & $\begin{array}{c}-0,109 * * * \\
(0,025)\end{array}$ & $\begin{array}{c}-0,235 * * * \\
(0,028)\end{array}$ \\
\hline Año 2017 & $\begin{array}{c}-0,167 * * * \\
(0,023)\end{array}$ & $\begin{array}{l}-0,020 \\
(0,024)\end{array}$ & $\begin{array}{c}-0,156 * * * \\
(0,027)\end{array}$ \\
\hline Trabaja en Barcelona & \multicolumn{3}{|c|}{ Categoría de referencia } \\
\hline Trabaja en Girona & & $\begin{array}{l}-0,045 \\
(0,039)\end{array}$ & $\begin{array}{l}-0,007 \\
(0,040)\end{array}$ \\
\hline Trabaja en Lleida & & $\begin{array}{c}0,002 \\
(0,041)\end{array}$ & $\begin{array}{l}-0,019 \\
(0,042)\end{array}$ \\
\hline Trabaja en Tarragona & & $\begin{array}{l}-0,074 \\
(0,048)\end{array}$ & $\begin{array}{l}-0,056 \\
(0,050)\end{array}$ \\
\hline Trabaja en otras CCAA españolas & & $\begin{array}{l}-0,051 \\
(0,035)\end{array}$ & $\begin{array}{l}-0,052 \\
(0,037)\end{array}$ \\
\hline Sector privado & & $\begin{array}{c}-0,259 * * * \\
(0,030)\end{array}$ & $\begin{array}{c}-0,226 * * * \\
(0,031)\end{array}$ \\
\hline Jornada a tiempo completo & & $\begin{array}{c}0,377 * * * \\
(0,024)\end{array}$ & $\begin{array}{c}0,350 * * * \\
(0,024)\end{array}$ \\
\hline
\end{tabular}

FUENTE: Elaboración propia a partir de las Encuestas de Inserción Laboral (AQU).

NOTA: Los errores estándar (en paréntesis) son robustos a la heterocedasticidad. * Significativo al 10 por 100 , ** Significativo al 5 por 100 . *** Significativo al 1 por 100 . 


\section{ANEXO 2 (Continuación) \\ ESTIMACIÓN DE LOS MODELOS PROBIT ORDENADO PARA EL DESAJUSTE EDUCATIVO}

\begin{tabular}{|c|c|c|c|}
\hline & (a) & (b) & (c) \\
\hline Contrato fijo/indefinido & \multicolumn{3}{|c|}{ Categoría de referencia } \\
\hline Trabajador autónomo & & $\begin{array}{c}0,147 * * * \\
(0,036)\end{array}$ & $\begin{array}{l}0,063^{*} \\
(0,038)\end{array}$ \\
\hline Contrato temporal & & $\begin{array}{c}-0,069 * * * \\
(0,022)\end{array}$ & $\begin{array}{c}-0,058 * * * \\
(0,022)\end{array}$ \\
\hline Becarios u otras situaciones & & $\begin{array}{c}-0,611 * * * \\
(0,097)\end{array}$ & $\begin{array}{c}-0,660 * * * \\
(0,102)\end{array}$ \\
\hline Antigüedad laboral & & $\begin{array}{c}0,056 * * * \\
(0,007)\end{array}$ & $\begin{array}{c}0,043 * * * \\
(0,007)\end{array}$ \\
\hline Empresa de hasta 10 trabajadores & \multicolumn{3}{|c|}{ Categoría de referencia } \\
\hline Empresa de 11-50 trabajadores & & $\begin{array}{c}0,141 * * * \\
(0,028)\end{array}$ & $\begin{array}{c}0,110 * * * \\
(0,029)\end{array}$ \\
\hline Empresa de 51-100 trabajadores & & $\begin{array}{c}0,225 * * * \\
(0,035)\end{array}$ & $\begin{array}{c}0,179 * * * \\
(0,036)\end{array}$ \\
\hline Empresa de 101-250 trabajadores & & $\begin{array}{c}0,115 * * * \\
(0,036)\end{array}$ & $\begin{array}{c}0,101 * * * \\
(0,037)\end{array}$ \\
\hline Empresa de 251-500 trabajadores & & $\begin{array}{c}0,161 * * * \\
(0,041)\end{array}$ & $\begin{array}{c}0,130 * * * \\
(0,042)\end{array}$ \\
\hline Empresa de más de 500 trabajadores & & $\begin{array}{c}0,113 * * * \\
(0,028)\end{array}$ & $\begin{array}{c}0,104 * * * \\
(0,029)\end{array}$ \\
\hline No información sobre el tamaño de la empresa & & $\begin{array}{c}0,027 \\
(0,058)\end{array}$ & $\begin{array}{c}0,048 \\
(0,059)\end{array}$ \\
\hline Agricultura, ganadería, silvicultura, caza & \multicolumn{3}{|c|}{ Categoría de referencia } \\
\hline $\begin{array}{l}\text { Pesca y piscicultura, acuicultura de aguas continen- } \\
\text { tales o marinas }\end{array}$ & & $\begin{array}{l}-0,043 \\
(0,481)\end{array}$ & $\begin{array}{l}-0,121 \\
(0,495)\end{array}$ \\
\hline Comb. sólidos, petróleo, gas y minerales radiactivos & & $\begin{array}{c}0,046 \\
(0,173)\end{array}$ & $\begin{array}{c}0,007 \\
(0,178)\end{array}$ \\
\hline $\begin{array}{l}\text { Electricidad, gas y agua. Fabricación de generadores } \\
\text { de vapor, captación,... de agua }\end{array}$ & & $\begin{array}{c}0,078 \\
(0,098)\end{array}$ & $\begin{array}{c}0,012 \\
(0,102)\end{array}$ \\
\hline Extracción y transformación de minerales & & $\begin{array}{c}0,522 \\
(0,333)\end{array}$ & $\begin{array}{c}0,467 \\
(0,337)\end{array}$ \\
\hline Industrias químicas & & $\begin{array}{c}0,094 \\
(0,101)\end{array}$ & $\begin{array}{c}0,013 \\
(0,105)\end{array}$ \\
\hline Industrias farmacéuticas y cosméticas & & $\begin{array}{c}0,238^{* *} * \\
(0,092)\end{array}$ & $\begin{array}{c}0,302 * * * \\
(0,095)\end{array}$ \\
\hline Metalurgia, material eléctrico y de precisión & & $\begin{array}{c}0,021 \\
(0,088)\end{array}$ & $\begin{array}{l}-0,000 \\
(0,091)\end{array}$ \\
\hline
\end{tabular}

FUENTE: Elaboración propia a partir de las Encuestas de Inserción Laboral (AQU).

NOTA: Los errores estándar (en paréntesis) son robustos a la heterocedasticidad. * Significativo al 10 por 100 , ** Significativo al 5 por 100. *** Significativo al 1 por 100. 


\section{ANEXO 2 (Continuación) \\ ESTIMACIÓN DE LOS MODELOS PROBIT ORDENADO PARA EL DESAJUSTE EDUCATIVO}

\begin{tabular}{|c|c|c|c|}
\hline & (a) & (b) & (c) \\
\hline Materiales de transporte. Fabricación vehículos mo- & & 0,061 & $-0,012$ \\
\hline Productos alimentarios, bebidas y tabaco & & $\begin{array}{c}0,014 \\
(0,090)\end{array}$ & $\begin{array}{l}-0,015 \\
(0,093)\end{array}$ \\
\hline Industrias textiles, del cuero y de confecciones & & $\begin{array}{c}-0,425 * * * \\
(0,108)\end{array}$ & $\begin{array}{c}-0,338 * * * \\
(0,112)\end{array}$ \\
\hline Industrias de la madera, corcho y muebles de madera & & $\begin{array}{c}-0,466^{* *} \\
(0,230)\end{array}$ & $\begin{array}{c}-0,549 * * \\
(0,240)\end{array}$ \\
\hline $\begin{array}{l}\text { Papel y artículos derivados. Artes gráficas y edición. } \\
\text { Fabricación de pasta de papel }\end{array}$ & & $\begin{array}{l}-0,086 \\
(0,120)\end{array}$ & $\begin{array}{l}-0,072 \\
(0,126)\end{array}$ \\
\hline $\begin{array}{l}\text { Caucho y plástico. Otras industrias manufactureras. } \\
\text { Reciclaje. Fabricación de vidrio }\end{array}$ & & $\begin{array}{l}-0,041 \\
(0,153)\end{array}$ & $\begin{array}{l}-0,091 \\
(0,160)\end{array}$ \\
\hline Construcción & & $\begin{array}{c}0,467 * * * \\
(0,090)\end{array}$ & $\begin{array}{c}0,430 * * * \\
(0,093)\end{array}$ \\
\hline Comercio y reparaciones & & $\begin{array}{c}-0,516 * * * \\
(0,085)\end{array}$ & $\begin{array}{c}-0,409 * * * \\
(0,088)\end{array}$ \\
\hline Restaurantes, cafés y hostelería & & $\begin{array}{c}-0,898 * * * \\
(0,098)\end{array}$ & $\begin{array}{c}-0,812 * * * \\
(0,101)\end{array}$ \\
\hline Transporte y actividades afines & & $\begin{array}{c}-0,326 * * * \\
(0,089)\end{array}$ & $\begin{array}{c}-0,277 * * * \\
(0,091)\end{array}$ \\
\hline Tecnologías de comunicación & & $\begin{array}{c}0,002 \\
(0,085)\end{array}$ & $\begin{array}{c}-0,154^{*} \\
(0,088)\end{array}$ \\
\hline $\begin{array}{l}\text { Medios de comunicación (radio, televisión, cine, } \\
\text { vídeo, editoriales...) }\end{array}$ & & $\begin{array}{r}0,250 * * \\
(0,097)\end{array}$ & $\begin{array}{c}0,114 \\
(0,100)\end{array}$ \\
\hline $\begin{array}{l}\text { Instituciones financieras, seguros y actividades inmo- } \\
\text { biliarias }\end{array}$ & & $\begin{array}{l}0,140^{*} \\
(0,084)\end{array}$ & $\begin{array}{c}0,211 * * \\
(0,087)\end{array}$ \\
\hline Servicios a las empresas. Alquiler de bienes & & $\begin{array}{c}0,364 * * * \\
(0,081)\end{array}$ & $\begin{array}{c}0,246^{* * * *} \\
(0,084)\end{array}$ \\
\hline Administración pública, defensa, y Seguridad Social & & $\begin{array}{c}-0,214 * * \\
(0,088)\end{array}$ & $\begin{array}{c}-0,363 * * * \\
(0,091)\end{array}$ \\
\hline Educación, investigación y servicios culturales & & $\begin{array}{c}0,651 * * * \\
(0,082)\end{array}$ & $\begin{array}{c}0,266 * * * \\
(0,089)\end{array}$ \\
\hline Sanidad y asistencia social & & $\begin{array}{c}0,544 * * * \\
(0,086)\end{array}$ & $\begin{array}{c}0,211 * * \\
(0,090)\end{array}$ \\
\hline Otras servicios prestados a la comunidad & & $\begin{array}{l}-0,093 \\
(0,089)\end{array}$ & $\begin{array}{c}-0,310 * * * \\
(0,093)\end{array}$ \\
\hline
\end{tabular}

FUENTE: Elaboración propia a partir de las Encuestas de Inserción Laboral (AQU).

NOTA: Los errores estándar (en paréntesis) son robustos a la heterocedasticidad. * Significativo al 10 por 100 , ** Significativo al 5 por 100 . *** Significativo al 1 por 100. 
ANEXO 2 (Continuación)

ESTIMACIÓN DE LOS MODELOS PROBIT ORDENADO PARA EL DESAJUSTE EDUCATIVO

\begin{tabular}{|c|c|c|c|}
\hline & (a) & (b) & (c) \\
\hline $\begin{array}{l}\text { Funciones de dirección } \\
\text { Funciones de comercio } \\
\text { Funciones de enseñanza } \\
\text { Funciones de asistencia } \\
\text { Funciones de I+D } \\
\text { Funciones de diseño } \\
\text { Funciones técnicas } \\
\text { Otras funciones cualificadas } \\
\text { Otras funciones no cualificadas }\end{array}$ & & & $\begin{array}{c}0,290 * * * \\
(0,022) \\
-0,257 * * * \\
(0,026) \\
0,547 * * * \\
(0,039) \\
0,608 * * * \\
(0,054) \\
0,506 * * * \\
(0,035) \\
-0,042 \\
(0,046) \\
0,379 * * * \\
(0,022) \\
-0,263 * * * \\
(0,029) \\
-0,919 * * * \\
(0,038)\end{array}$ \\
\hline $\begin{array}{l}\text { cut } 1 \\
\text { cut } 2 \\
\text { cut } 3\end{array}$ & $\begin{array}{c}-2,280 * * * \\
(0,314) \\
-2,099 * * * \\
(0,314) \\
-1,905 * * * \\
(0,314) \\
\end{array}$ & $\begin{array}{c}-1,884 * * * \\
(0,352) \\
-1,679 * * * \\
(0,352) \\
-1,466 * * * \\
(0,352) \\
\end{array}$ & $\begin{array}{c}-2,167 * * * \\
(0,347) \\
-1,940 * * * \\
(0,347) \\
-1,708 * * * \\
(0,347) \\
\end{array}$ \\
\hline Número de observaciones & 30.265 & 30.265 & 30.265 \\
\hline
\end{tabular}

FUENTE: Elaboración propia a partir de las Encuestas de Inserción Laboral (AQU).

NOTA: Los errores estándar (en paréntesis) son robustos a la heterocedasticidad. * Significativo al 10 por 100 , ** Significativo al 5 por 100 . *** Significativo al 1 por 100. 